TRANSACTIONS OF THE

AMERICAN MATHEMATICAL SOCIETY

Volume 362, Number 1, January 2010, Pages 199-227

S 0002-9947(09)04962-9

Article electronically published on July 30, 2009

\title{
DIRICHLET SERIES FOR FINITE COMBINATORIAL RANK DYNAMICS
}

\author{
G. EVEREST, R. MILES, S. STEVENS, AND T. WARD
}

\begin{abstract}
We introduce a class of group endomorphisms - those of finite combinatorial rank - exhibiting slow orbit growth. An associated Dirichlet series is used to obtain an exact orbit counting formula, and in the connected case this series is shown to to be a rational function of exponential variables. Analytic properties of the Dirichlet series are related to orbit-growth asymptotics: depending on the location of the abscissa of convergence and the degree of the pole there, various orbit-growth asymptotics are found, all of which are polynomially bounded.
\end{abstract}

\section{INTRODUCTION}

A closed orbit $\tau$ of length $|\tau|=n$ for a map $\alpha: X \rightarrow X$ is a set of the form

$$
\left\{x, \alpha(x), \alpha^{2}(x), \ldots, \alpha^{n}(x)=x\right\}
$$

with cardinality $n$ (in our setting $X$ will always be a compact metric space and $\alpha$ a continuous map). Dynamical analogues of the prime number theorem concern the asymptotic behavior of quantities such as

$$
\pi_{\alpha}(N)=\mid\{\tau \text { a closed orbit of } \alpha|| \tau \mid \leqslant N\} \mid .
$$

When $X$ has a metric structure with respect to which $\alpha$ is hyperbolic, results of Parry and Pollicott [11, [12, [13] and others apply to give a precise understanding of the growth properties of $\pi_{\alpha}$. Without hyperbolicity less is known: Waddington [17] considered the case of a quasihyperbolic toral automorphism, and the authors [3] found asymptotics for connected $S$-integer systems with $S$ finite (these are isometric extensions of hyperbolic automorphisms of arithmetic origin).

Writing

$$
\mathrm{F}_{\alpha}(n)=\left|\left\{x \in X \mid \alpha^{n} x=x\right\}\right|
$$

for the number of points fixed by $\alpha^{n}$, the dynamical zeta function of $\alpha$ is defined by

$$
\zeta_{\alpha}(z)=\exp \sum_{n=1}^{\infty} \frac{z^{n}}{n} \mathrm{~F}_{\alpha}(n),
$$

which has a formal expansion as an Euler product,

$$
\zeta_{\alpha}(z)=\prod_{\tau}\left(1-z^{|\tau|}\right)^{-1}
$$

Received by the editors July 25, 2007.

2000 Mathematics Subject Classification. Primary 37C30; Secondary 26E30, 12J25.

This research was supported by E.P.S.R.C. grant EP/C015754/1.

(C)2009 American Mathematical Society Reverts to public domain 28 years from publication 
where the product is taken over all closed orbits of $\alpha$. In the hyperbolic and quasihyperbolic settings, the dynamical zeta function is either rational or has a meromorphic extension beyond its radius of convergence, allowing analytic methods to be used to obtain asymptotic estimates for $\pi_{\alpha}$. For $S$-integer systems with $S$ finite the dynamical zeta function typically has a natural boundary, so more direct methods have to be used in [3]. In all these cases the asymptotic takes the form

$$
\pi_{\alpha}(N) \sim \frac{e^{h(\alpha) N}}{N} \Psi(N)
$$

where $\Psi$ is an explicit almost-periodic function bounded away from zero and infinity, constant in the hyperbolic cases, and $h(\alpha)$ is the topological entropy of $\alpha$. The $S$-integer systems with $S$ finite may be viewed as arithmetical perturbations of (quasi)hyperbolic systems, and the orbit growth is still essentially exponential.

Our purpose here is to describe orbit growth for a class of dynamical systems much further from hyperbolicity. We consider canonical partially ordered sets of sequences of periodic point counts for which hyperbolic systems produce maximal elements (see Example 2.4). Our interest lies at the other extreme, with systems that produce elements with finite rank in the poset (see Definition 2.9). These systems are found to possess an orbit structure which allows Dirichlet series to be used directly. The simplest non-trivial example of a group automorphism with finite combinatorial rank is the automorphism $\alpha$ dual to the map $x \mapsto 2 x$ on the localization $\mathbb{Z}_{(3)}$ of the integers at the prime 3 (the periodic point data for this map is the same as that of the map $z \mapsto z^{2}$ on $\left\{z \in \mathbb{C} \mid z^{3^{k}}=1\right.$ for some $\left.\left.k \geqslant 0\right\}\right)$. By [2], this system has $\mathrm{F}_{\alpha}(n)=\left|2^{n}-1\right|_{3}^{-1}$, and Stangoe [15] used this to show the asymptotic

$$
\pi_{\alpha}(N)=\frac{1}{\log 3} \log (N)+\mathrm{O}(1)
$$

for all $N \geqslant 1$. In some cases, this distance from the hyperbolic case can be expressed geometrically: for systems with an adelic covering space (see [2] or [7]) the systems studied here have infinitely many $p$-adic eigendirections in which they behave like isometries, and these places cover all but finitely many of the available places.

The main results concern an algebraic system $(X, \alpha)$ of finite combinatorial rank and the number $\mathrm{O}_{\alpha}(n)$ of closed orbits of length $n$ under $\alpha$. We associate the Dirichlet series

$$
\mathrm{d}_{\alpha}(z)=\sum_{n=1}^{\infty} \frac{\mathrm{O}_{\alpha}(n)}{n^{z}}
$$

to $(X, \alpha)$ and use this to study the orbit-growth function

$$
\pi_{\alpha}(N)=\sum_{n \leqslant N} \mathrm{O}_{\alpha}(n)
$$

In Theorem 3.3 we find an exact expression for the Dirichlet series and use this to show that, in the connected case, there is a finite set of positive integers $\mathcal{C}$ with the property that $\mathrm{d}_{\alpha}(z)$ is a rational function of the variables $\left\{c^{-z} \mid c \in \mathcal{C}\right\}$ (see Example 4.1 for a simple instance of this rationality result).

The asymptotic behaviour of $\pi_{\alpha}$ is governed by the abscissa of convergence $\sigma$ of $\mathrm{d}_{\alpha}$ and the order $K$ of the pole at $\sigma$. For the case $\sigma=0$, Theorem 3.6)(a) shows that

$$
\pi_{\alpha}(N)=C(\log N)^{K}+\mathrm{O}\left((\log N)^{K-1}\right)
$$


Example 7.2 illustrates this result. For $\sigma>0$ the situation is more involved. In combinatorial rank one (in which case the pole at $\sigma$ is necessarily simple), Theorem 3.5. shows that

$$
\pi_{\alpha}(N)=\delta(N) N^{\sigma}+\mathrm{O}(1),
$$

where $\delta$ is an explicit oscillatory function bounded away from zero and infinity. An instance of this phenomenon may be found in Example 4.3

For the general case of a simple pole, we show in Theorem 3.6(b) a Chebychev result of the form

$$
A N^{\sigma} \leqslant \pi_{\alpha}(N) \leqslant B N^{\sigma}
$$

for constants $A, B>0$. Given the oscillatory function that arises in combinatorial rank one, no stronger asymptotic can be expected.

Finally, and surprisingly, in the case $\sigma>0$ and $K \geqslant 2$, Theorem 3.6(c) gives the exact asymptotic

$$
\pi_{\alpha}(N) \sim C N^{\sigma}(\log N)^{K-1}
$$

This comes about because the higher-order pole introduces a factor which randomizes the oscillatory behaviour seen in the case of a simple pole. This is most easily seen in Example 7.4.

Typically, particularly in analytic number theory, the route from the singular behaviour of a Dirichlet series to a counting function goes via a Tauberian theorem. However, the presence of singularities of $d_{\alpha}$ arbitrarily close together along the line $\Re(z)=\sigma$ hampers this approach. In simpler situations such as in Example 4.1 (where the singularites on the line $\Re(z)=\sigma$ are equally spaced), Agmon's Tauberian theorem [1] or Perron's Theorem [5. Th. 13] can be applied. However, even in these simpler cases, it is easier to obtain asymptotics directly from the series expression for $\mathrm{d}_{\alpha}$.

The letter $C$ is used to denote various constants independent of the variables $n$, $N, x$ and so on; $\mathbb{N}_{0}$ denotes $\mathbb{N} \cup\{0\}$.

\section{Finite COMBinatorial RANK}

Let $X$ be a compact metrizable abelian group of finite topological dimension, and let $\alpha$ be a continuous ergodic epimorphism of $X$ with finite topological entropy. Let $\mathcal{D}$ denote the class of such dynamical systems $(X, \alpha)$. For $(X, \alpha) \in \mathcal{D}$, Lemma 2.1 below will show that $\mathrm{F}_{\alpha}(n)$ is finite for all $n \geqslant 1$.

Consider the ring $\mathbb{Z}^{\mathbb{N}}$ with the product ordering $\preccurlyeq\left(\right.$ so $\left(a_{n}\right) \preccurlyeq\left(b_{n}\right)$ if $a_{n} \leqslant b_{n}$ for all $n \in \mathbb{Z}$ ). For a subset $A \subset \mathbb{Z}^{\mathbb{N}}, a \in A$ is said to have finite rank in $A$ if there is some $C>0$ such that all strictly decreasing chains of elements of $A$ starting at $a$ have length at most $C$.

Pontryagin duality gives a one-to-one correspondence between elements of $\mathcal{D}$ and certain countable modules over the domain $R=\mathbb{Z}[t]$ as follows. The additive group $M=\widehat{X}$ has the structure of an $R$-module by identifying $x \mapsto t x$ with the map $\widehat{\alpha}$ and extending in an obvious way to polynomials. The module $M$ satisfies the following conditions:

- $M$ is countable (since $X$ is metrizable);

- the map $x \mapsto t x$ is a monomorphism of $M$ (since $\alpha$ is onto);

- the set of associated primes $\operatorname{Ass}(M)$ is finite and consists entirely of nonzero principal ideals, none of which is generated by cyclotomic polynomials (since $X$ is finite-dimensional, $\alpha$ is ergodic and $h(\alpha)<\infty$ ); 
- for each $\mathfrak{p} \in \operatorname{Ass}(M)$,

$$
m(\mathfrak{p})=\operatorname{dim}_{\mathbb{K}(\mathfrak{p})} M_{\mathfrak{p}}<\infty,
$$

where $\mathbb{K}(\mathfrak{p})$ denotes the field of fractions of $R / \mathfrak{p}$ (since $X$ is finite-dimensional and $h(\alpha)<\infty)$.

Conversely, if $M$ is an $R$-module satisfying these four properties and $\alpha_{M}$ denotes the epimorphism of $X_{M}=\widehat{M}$ dual to $x \mapsto t x$ on $M$, then

$$
\left(X_{M}, \alpha_{M}\right) \in \mathcal{D} \text {. }
$$

Denote the class of all such $R$-modules $M$ by $\widehat{\mathcal{D}}$.

The next lemma gives a formula for the number of periodic points of an element of $\mathcal{D}$ in terms of valuations of sequences of the form

$$
\theta(\mathfrak{p})=\left(\bar{t}^{n}-1\right)_{n \geqslant 1},
$$

where $\bar{t}$ denotes the image of $t$ in $R / \mathfrak{p}$ for some prime ideal $\mathfrak{p}$ associated to $M$. In order to describe this, suppose that $\mathfrak{p} \subset R$ is a principal prime ideal so that $\mathbb{K}(\mathfrak{p})$ is a global field with finite residue class fields. Let $P(\mathfrak{p}), \mathrm{P}_{0}(\mathfrak{p}), \mathrm{P}_{\infty}(\mathfrak{p})$ denote the places, finite places, and infinite places of $\mathbb{K}(\mathfrak{p})$, respectively. In what follows, if $v$ denotes a normalized additive valuation corresponding to a place in $P_{0}(\mathfrak{p})$, then $|\cdot|_{v}$ is defined by $|\cdot|_{v}=\left|\mathfrak{K}_{v}\right|^{-v(\cdot)}$, where $\mathfrak{K}_{v}$ is the residue class field. For ease of notation, if $P$ is any subset of the set of places of a global field $K$, then write

$$
|x|_{P}=\prod_{v \in P}|x|_{v}
$$

for $x \in K$, with the convention that $|x|_{\varnothing}=1$. Finally, note that each $v \in \mathrm{P}_{0}(\mathfrak{p})$ also induces a map $|\cdot|_{v}: \mathbb{K}(\mathfrak{p})^{\mathbb{N}} \rightarrow \mathbb{Q}^{\mathbb{N}}$, and $|\theta(\mathfrak{p})|_{v}$ is a unit in $\mathbb{Q}^{\mathbb{N}}$ provided that $\mathfrak{p}$ is not generated by a cyclotomic polynomial.

Write $\mathrm{F}_{\alpha}$ for the sequence $\left(\mathrm{F}_{\alpha}(n)\right)_{n \geqslant 1}$; for sequences $a=\left(a_{n}\right)_{n \geqslant 1}$ and $b=\left(b_{n}\right)_{n \geqslant 1}$ write $a b, a^{k}$ for the sequences $\left(a_{n} b_{n}\right)_{n \geqslant 1},\left(a_{n}^{k}\right)_{n \geqslant 1}$, respectively.

Lemma 2.1. Let $M \in \widehat{\mathcal{D}}$. Then there exist $\mathfrak{p}_{1}, \ldots, \mathfrak{p}_{r} \in \operatorname{Ass}(M)$, and sets of places $P_{1}, \ldots, P_{r}$ with $P_{i} \subset \mathrm{P}_{0}\left(\mathfrak{p}_{i}\right), 1 \leqslant i \leqslant r$, such that

$$
\mathrm{F}_{\alpha_{M}}=\prod_{i=1}^{r}\left|\theta\left(\mathfrak{p}_{i}\right)\right|_{P_{i}}^{-1} .
$$

Proof. This is shown in [9, Th. 1.1].

For $M \in \widehat{\mathcal{D}}$, set

$$
[M]=\left\{\mathrm{F}_{\alpha_{L}} \mid L \text { is a submodule of } M\right\} \subset \mathbb{Z}^{\mathbb{N}} .
$$

Lemma 2.1 and the next result together show how $[M]$ relates to the simpler constituent sets of sequences of the form $[\mathbb{K}(\mathfrak{p})]$. The members of a set such as $[\mathbb{K}(\mathfrak{p})]$ are sequences of periodic points for $S$-integer systems (see [2]). As usual write $2^{P}$ for the set of subsets of $P$.

Lemma 2.2. Let $\mathfrak{p} \subset R$ be a principal prime ideal which is not generated by a cyclotomic polynomial, and set

$$
P=\left\{v \in \mathrm{P}_{0}(\mathfrak{p})|| R /\left.\mathfrak{p}\right|_{v} \text { is bounded }\right\} .
$$

Then $[\mathbb{K}(\mathfrak{p})]$ is the image of the map $\psi: 2^{P} \rightarrow \mathbb{Z}^{\mathbb{N}}$, where

$$
\psi(Q)=|\theta(\mathfrak{p})|_{Q}^{-1} .
$$


Proof. The proof of $\left[9\right.$, Th. 1.1] shows that $[\mathbb{K}(\mathfrak{p})] \subset \psi\left(2^{P}\right)$, so it remains to show the reverse inclusion. Fix a subset $Q \subset P$. If $Q=\varnothing$, set $L=\mathbb{K}(\mathfrak{p})$; otherwise set $L=\bigcap_{v \in Q} R_{v}$, where $R_{v}$ is the discrete valuation ring of $\mathbb{K}(\mathfrak{p})$ corresponding to $v$. Then $L$ is a submodule of $\mathbb{K}(\mathfrak{p})$ with $\mathrm{F}_{\alpha_{L}}=\psi(Q)$ by construction.

It follows that

$$
[M] \subset \prod_{\mathfrak{p} \in \operatorname{Ass}(M)}[\mathbb{K}(\mathfrak{p})]^{m(\mathfrak{p})},
$$

and each $[\mathbb{K}(\mathfrak{p})]$ has a description as in Lemma 2.2 .

Proposition 2.3. For any $R$-module $M \in \widehat{\mathcal{D}}$ the following holds.

(a) For each $\mathfrak{p} \in \operatorname{Ass}(M)$, the set $[\mathbb{K}(\mathfrak{p})]$ contains a greatest element

$$
s(\mathfrak{p})=\mathrm{F}_{\alpha_{R / \mathfrak{p}}} .
$$

Furthermore, for any $a \in[\mathbb{K}(\mathfrak{p})], a^{-1} s(\mathfrak{p}) \in[\mathbb{K}(\mathfrak{p})]$.

(b) The set $[M]$ contains a greatest element

$$
\mathrm{F}_{\alpha_{L}}=\prod_{\mathfrak{p} \in \operatorname{Ass}(M)} s(\mathfrak{p})^{m(\mathfrak{p})}
$$

given by a Noetherian submodule $L \subset M$.

Proof. (a) This follows from the description given by Lemma 2.2, The sequence given by $\psi(P)=\mathrm{F}_{\alpha_{R / \mathfrak{p}}}$ is the greatest element of $[\mathbb{K}(\mathfrak{p})]$ and for any subset $Q \subset P$, we have $\psi(Q) \psi(P \backslash Q)=\psi(P)$.

(b) Let

$$
s=\prod_{\mathfrak{p} \in \operatorname{Ass}(M)} s(\mathfrak{p})^{m(\mathfrak{p})} .
$$

Then (a) and Lemma 2.1 show that $\mathrm{F}_{\alpha_{M}} \preccurlyeq s$. The result follows by observing that there is a Noetherian submodule $L \subset M$ with a prime filtration in which each of the primes $\mathfrak{p} \in \operatorname{Ass}(M)$ appears with multiplicity $m(\mathfrak{p})$ and no other primes appear. It follows that $\mathrm{F}_{\alpha_{L}}=s$.

Example 2.4. If $\alpha_{M}$ is a quasihyperbolic toral epimorphism, then $F_{\alpha_{M}}$ is the greatest element of $[M]$. More generally, for any Noetherian module $M \in \widehat{\mathcal{D}}, \mathrm{F}_{\alpha_{M}}$ is the greatest element of $[M]$.

The next result gives a convenient characterization of the finite-rank sequences in a poset of the form $[\mathbb{K}(\mathfrak{p})]$; this will be useful when considering dynamical systems of finite combinatorial rank in the next section.

Theorem 2.5. Let $\mathfrak{p} \subset R$ be a principal prime ideal which is not generated by a cyclotomic polynomial, and let $a \in[\mathbb{K}(\mathfrak{p})]$. Then a has finite rank if and only if there is a finite set of places $Q \subset \mathrm{P}_{0}(\mathfrak{p})$ such that $|\theta(\mathfrak{p})|_{Q}^{-1}=a$.

The proof of Theorem 2.5 requires the following lemma, taken from [8, Lem. 4.9]. Recall that $\mathfrak{K}_{v}$ denotes the residue class field of a place $v$, write $\ell_{v}$ for the multiplicative order of the image of $t$ in $\mathfrak{K}_{v}^{\times}$and write

$$
r_{v}=\left[\mathfrak{K}_{v}: \mathbb{F}_{p}\right] \times v(p),
$$

where $p=\operatorname{char}\left(\mathfrak{K}_{v}\right)$. 
Lemma 2.6. Let $\mathfrak{p} \subset R$ be a principal prime ideal not generated by a cyclotomic polynomial, let $v \in \mathrm{P}_{0}(\mathfrak{p})$ have the property that $|\bar{t}|_{v}=1$, and let $p=\operatorname{char}\left(\mathfrak{K}_{v}\right)$. Then there are constants $D \geqslant 1, E \geqslant 0$ such that

$$
\left|\theta(\mathfrak{p})_{n}\right|_{v}^{-1}= \begin{cases}1 & \text { if } \ell_{v} \nmid n, \\ D p^{r_{v}} \operatorname{ord}_{p}(n) & \text { if } \ell_{v} \mid n, \operatorname{char}(\mathbb{K}(\mathfrak{p}))=0 \text { and } \operatorname{ord}_{p}(n) \geqslant E, \\ \left|\theta(\mathfrak{p})_{\ell_{v}}\right|_{v}^{-|n|_{p}^{-1}} & \text { if } \ell_{v} \mid n \text { and } \operatorname{char}(\mathbb{K}(\mathfrak{p}))>0 .\end{cases}
$$

Proof of Theorem 2.5, Let $\mathbb{K}=\mathbb{K}(\mathfrak{p}), \theta=\theta(\mathfrak{p})$ and let $P, \psi$ be as in Lemma 2.2, For any $Q \subset P$, set

$$
\widetilde{Q}=\left\{\left.v \in Q|| \theta\right|_{v} ^{-1} \neq 1\right\} .
$$

Notice that if $|\bar{t}|_{v}<1$, then $|\theta|_{v}=1$, so $|\bar{t}|_{v}=1$ for all $v \in \widetilde{P}$.

Suppose that $a$ has finite combinatorial rank and that $Q \subset P$ satisfies $\psi(Q)=a$. Assume $\widetilde{Q}$ is infinite. By choosing a strictly decreasing chain

$$
\widetilde{Q}=Q_{1} \supset Q_{2} \supset \cdots,
$$

it follows that

$$
a=\psi\left(Q_{1}\right) \succ \psi\left(Q_{2}\right) \succ \cdots,
$$

contradicting the assumption that $a$ has finite combinatorial rank. Hence $\widetilde{Q}$ is finite and satisfies $\psi(\widetilde{Q})=a$.

Conversely, suppose there is a finite set $Q \subset P$ with $\psi(Q)=a$. Without loss of generality, assume that $Q=\widetilde{Q}$. If there are only finitely many distinct $Q^{\prime} \subset \widetilde{P}$ with $\psi\left(Q^{\prime}\right) \preccurlyeq a$, the required result follows. Hence, for a contradiction assume there are infinitely many such $Q^{\prime}$.

First suppose that $\operatorname{char}(\mathbb{K})=0$. Then the set of rational primes

$$
S=\left\{\operatorname{char}\left(\mathfrak{K}_{v}\right) \mid v \in Q\right\}
$$

is finite. Furthermore, using Lemma 2.6 there exist positive constants $c, D, E$ such that for each $n \geqslant 1$ with $\min _{p \in S}\left\{\operatorname{ord}_{p}(n)\right\} \geqslant E$,

$$
a_{n}=\left|\theta_{n}\right|_{Q}^{-1} \leqslant D c^{j(n)},
$$

where $j(n)=\max \left\{\operatorname{ord}_{p}(n) \mid p \in S\right\}$.

There are only finitely many $v \in \mathrm{P}_{0}(\mathbb{K})$ with $\operatorname{char}\left(\mathfrak{K}_{v}\right)$ equal to a given prime. So, since there are infinitely many distinct $Q^{\prime} \subset \widetilde{P}$ with $\psi\left(Q^{\prime}\right) \preccurlyeq a$, it is possible to choose $Q^{\prime}$ to contain a place $v$ with $\operatorname{char}\left(\mathfrak{K}_{v}\right) \notin S$. For $r \geqslant 0$, set

$$
n(r)=\ell_{v} q^{r} \prod_{p \in S} p^{E}
$$

where $q=\operatorname{char}\left(\mathfrak{K}_{v}\right)$. Since $j(n(r))$ is bounded, (2.4) shows that $a_{n(r)}$ is also bounded. However, again applying Lemma 2.6, for a sufficiently large choice of $r$,

$$
\left|\theta_{n(r)}\right|_{Q^{\prime}}^{-1} \geqslant\left|\theta_{n(r)}\right|_{v}^{-1}>a_{n(r)},
$$

contradicting $\psi\left(Q^{\prime}\right) \preccurlyeq a$.

Now assume that $\operatorname{char}(\mathbb{K})=p>0$. Then $R / \mathfrak{p}=\mathbb{F}_{p}[\bar{t}]$ and $\bar{t}$ is transcendental over $\mathbb{F}_{p}$. Since $Q$ is finite, Lemma 2.6] shows that the set $\left\{a_{n} \mid \operatorname{ord}_{p}(n)=0, n \geqslant 1\right\}$ is bounded. There are, by assumption, infinitely many distinct $Q^{\prime} \subset \widetilde{P}$ with $\psi\left(Q^{\prime}\right) \preccurlyeq$ $a$, so it is possible to choose such a $Q^{\prime}$ to contain a place $v$ for which $\left|\mathfrak{K}_{v}\right|$ is larger 
than this bound. However, $\left|\theta_{\ell_{v}}\right|_{v} \leqslant\left|\mathfrak{K}_{v}\right|^{-1}$ and since $\operatorname{ord}_{p}\left(\ell_{v}\right)=0$,

$$
\left|\theta_{\ell_{v}}\right|_{Q^{\prime}}^{-1} \geqslant\left|\theta_{\ell_{v}}\right|_{v}^{-1} \geqslant\left|\mathfrak{K}_{v}\right|>a_{\ell_{v}}
$$

contradicting $\psi\left(Q^{\prime}\right) \preccurlyeq a$ again.

$1,3,7,15,31,63,127,255,511,1023$

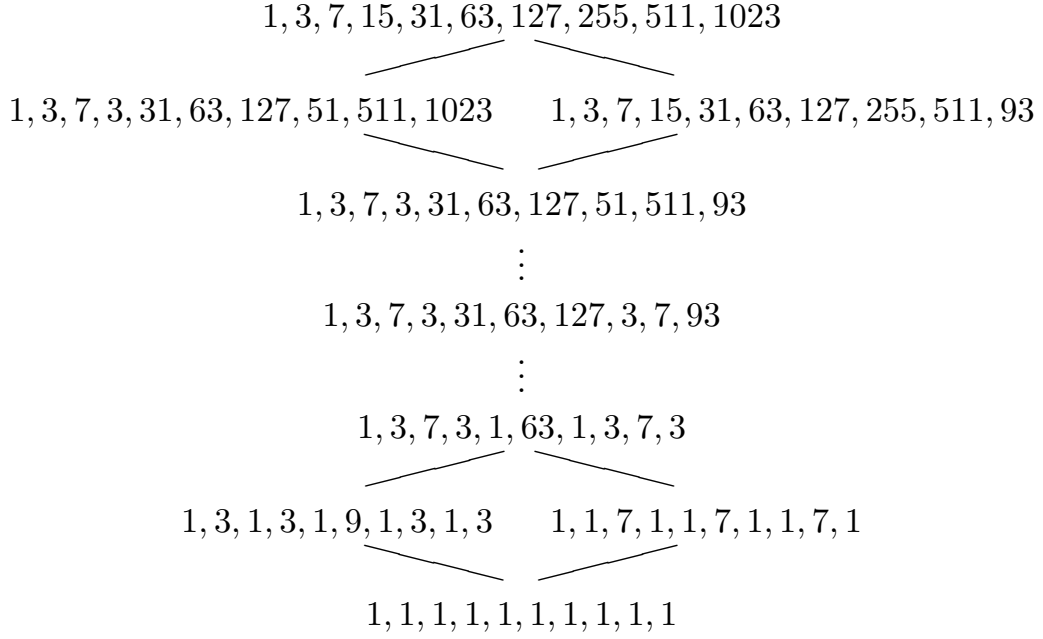

Figure 1. Part of the Hasse diagram for $[\mathbb{K}(t-2)]$

Example 2.7. The poset $[\mathbb{K}(t-2)]$, which contains the sequences of periodic point counts arising from algebraic factors of the map $x \mapsto 2 x$ on the solenoid $\widehat{\mathbb{Q}}$, is illustrated in Figure 1 (the figure shows a small part of the full poset: the first level from the bottom has infinitely many sequences, parameterised by the rational primes). The greatest element corresponds to the circle doubling map $x \mapsto 2 x$ on $\mathbb{T}=\mathbb{R} / \mathbb{Z}$, and the central sequence represents $\psi(Q)$, where $Q$ is the set of Mersenne primes. According to Theorem 2.5, $\psi(Q)$ has finite rank if and only if there are finitely many Mersenne primes. The least element corresponds to the map $x \mapsto 2 x$ on $\widehat{\mathbb{Q}}$, corresponding to $\psi(Q)$ where $Q$ is the set of all rational primes.

Remark 2.8. The $S$-integer systems studied by Chothi, Everest and Ward [2] give an alternative way to describe the periodic point sequences we study. In the connected case, they may be described as follows. Fix an algebraic number field $\mathbb{K}$ with set of places $\mathrm{P}(\mathbb{K})$ and set of infinite places $\mathrm{P}_{\infty}(\mathbb{K})$, an element of infinite multiplicative order $\xi \in \mathbb{K}^{*}$, and a set $S \subset \mathrm{P}(\mathbb{K}) \backslash \mathrm{P}_{\infty}(\mathbb{K})$ with the property that $|\xi|_{w} \leqslant 1$ for all $w \notin S \cup \mathrm{P}_{\infty}(\mathbb{K})$. The associated ring of $S$-integers is

$$
R_{S}=\left\{\left.x \in \mathbb{K}|| x\right|_{w} \leqslant 1 \text { for all } w \notin S \cup \mathrm{P}_{\infty}(\mathbb{K})\right\} .
$$

Let $X$ be the character group of $R_{S}$, and define an endomorphism $\alpha$ to be the dual of the map $x \mapsto \xi x$ on $R_{S}$. By [2, Lemma 5.2] the number of points in $X$ fixed by $\alpha^{n}$ is

$$
\mathrm{F}_{\alpha}(n)=\prod_{w \in S \cup \mathrm{P}_{\infty}(\mathbb{K})}\left|\xi^{n}-1\right|_{w}
$$

In the language of 2], the systems we are interested in are the co-finite ones (those for which $\mathrm{P}(\mathbb{K}) \backslash S$ is a finite set) and finite products of them. Using the product 
formula for global fields, the periodic point formula when $S$ is co-finite reduces to one of the factors in (2.1).

In view of the canonical inclusion (2.2) and the results of this section, we make the following definition.

Definition 2.9. Let $M \in \widehat{\mathcal{D}}$. Then $\alpha_{M}$ has finite combinatorial rank if $\mathrm{F}_{\alpha_{M}}$ has finite rank in

$$
\prod_{\mathfrak{p} \in \operatorname{Ass}(M)}[\mathbb{K}(\mathfrak{p})]^{m(\mathfrak{p})} .
$$

For the rest of the paper, we assume that $(X, \alpha) \in \mathcal{D}$.

\section{Dynamical Dirichlet SeRies}

Writing $\mathrm{O}_{\alpha}(n)$ for the number of orbits of length $n$ under $\alpha$, we have the following arithmetical relation between periodic points and orbits:

$$
\mathrm{F}_{\alpha}(n)=\sum_{d \mid n} d \mathrm{O}_{\alpha}(d)
$$

By Möbius inversion,

$$
\mathrm{O}_{\alpha}(n)=\frac{1}{n} \sum_{d \mid n} \mu(n / d) \mathrm{F}_{\alpha}(d)
$$

Definition 3.1. The dynamical Dirichlet series associated to the map $\alpha$ is the formal series

$$
\mathrm{d}_{\alpha}(z)=\sum_{n=1}^{\infty} \frac{\mathrm{O}_{\alpha}(n)}{n^{z}} .
$$

Example 3.2. The examples we wish to study are group epimorphisms of finite combinatorial rank, but the quadratic map $\alpha: x \mapsto 1-c x^{2}$ on the interval $[-1,1]$ at the Feigenbaum value $c=1.401155 \cdots$ (see Ruelle's survey [14]) gives a particularly simple example of a dynamical Dirichlet series. This map has

$$
\mathrm{O}_{\alpha}(n)= \begin{cases}1 & \text { if } n=2^{k} \text { for some } k \geqslant 0 \\ 0 & \text { if not, }\end{cases}
$$

so $\mathrm{d}(z)=\frac{1}{1-2^{-z}}$. In this example it is clear that $\pi_{\alpha}(N)=\frac{\log N}{\log 2}+\mathrm{O}(1)$ in accordance with Theorem 3.6. but it is important to emphasise that in general the asymptotic growth statements cannot be deduced from the analytic behaviour of the Dirichlet series alone.

Using convolution of Dirichlet series (see [16, Sec. 3.7]), an alternative way of expressing the relation (3.1) is

$$
\mathrm{d}_{\alpha}(z)=\frac{1}{\zeta(z+1)} \sum_{n=1}^{\infty} \frac{\mathrm{F}_{\alpha}(n) / n}{n^{z}},
$$

where as usual

$$
\zeta(z+1)=\sum_{n=1}^{\infty} \frac{1 / n}{n^{z}} .
$$

For systems of finite combinatorial rank, this observation is extremely useful: it is possible to extract $\zeta(z+1)$ as a factor of the series on the right-hand side of (3.2), 
resulting in an exact expression for orbit counting. Note that only combinatorial (rather than analytic) properties of the zeta function are of importance here.

Recall that the set of rational primes $p$ with

$$
p \mid \mathrm{F}_{\alpha}(n) \text { for some } n \in \mathbb{N}
$$

is finite when $F_{\alpha}$ has finite rank.

Theorem 3.3. Let $(X, \alpha)$ be a system of finite combinatorial rank and let $Q$ be the set of rational primes given by (3.3). Then $\mathrm{d}_{\alpha}(z)$ is a linear combination of Dirichlet series of the form

$$
\sum_{\mathbf{e} \in \mathbb{N}_{0}^{P}} \frac{\varphi_{P}(\Lambda(\mathbf{e}))}{\left(b \varphi_{P}(\mathbf{e})\right)^{z}}
$$

where $b \in \mathbb{N}, P \subset Q, \Lambda: \mathbb{N}_{0}^{P} \rightarrow \mathbb{N}_{0}^{P}$ and $\varphi_{P}(\mathbf{e})=\prod_{p \in P} p^{e_{p}}$.

Furthermore, all these quantities can be determined explicitly. In particular, denoting the local field corresponding to a place $v$ by $\mathbb{K}_{v}$,

$$
\Lambda(\mathbf{e})_{p}=-e_{p}+\sum_{v \in S(p)} r_{v} \lambda_{v}\left(e_{p}\right)
$$

where $S(p)$ is a set of places with residue class fields of characteristic $p, r_{v}$ is given by (2.3), and

$$
\lambda_{v}\left(e_{p}\right)= \begin{cases}e_{p} & \text { if } \operatorname{char}\left(\mathbb{K}_{v}\right)=0, \\ \eta_{v} p^{e_{p}} & \text { if } \operatorname{char}\left(\mathbb{K}_{v}\right)>0,\end{cases}
$$

for some positive integer constant $\eta_{v}$.

Theorem 3.3 and its proof allow us to deduce the following result for connected systems.

Theorem 3.4. Let $(X, \alpha)$ be a connected system of finite combinatorial rank. Then there is a finite set $\mathcal{C} \subset \mathbb{N}$ with the property that $\mathrm{d}_{\alpha}(z)$ is a rational function of the variables $\left\{c^{-z} \mid c \in \mathcal{C}\right\}$.

In principle, Theorem 3.3 provides an exact formula for orbit counting, since it implies that $\pi_{\alpha}(N)$ is a linear combination of expressions of the form

$$
\sum_{\substack{\mathbf{e} \in \mathbb{N}_{0}^{P}, \varphi_{P}(\mathbf{e}) \leqslant N / b}} \varphi_{P}(\Lambda(\mathbf{e})) .
$$

However, a closed asymptotic expression involving elementary functions is often more desirable, the prime number theorem being a case in point. Our focus is on connected systems, although Theorem 3.3 may also be applied in the disconnected case (cf. Example 4.4).

Theorem 3.5. Let $(X, \alpha)$ be a connected system of combinatorial rank one. Then

$$
\pi_{\alpha}(N)= \begin{cases}C \log N+\mathrm{O}(1) & \text { if } r_{v}=1 \\ \delta(N) N^{r_{v}-1}+\mathrm{O}(1) & \text { otherwise }\end{cases}
$$

where $\delta(N)$ is an explicit oscillatory function bounded away from zero and infinity, $v$ is the associated place, and $r_{v}$ is given by (2.3). 
Theorem 3.5 shows that an elementary asymptotic formula for $\pi_{\alpha}$ cannot be expected in general. Typically, an oscillatory function similar to that in Theorem 3.5 appears when the abscissa of convergence of $d_{\alpha}$ is greater than zero and is a simple pole; we provide a Chebychev result in this case. In all other cases, an elementary asymptotic formula is found.

Theorem 3.6. Let $(X, \alpha)$ be a connected system of finite combinatorial rank whose Dirichlet series $\mathrm{d}_{\alpha}$ has abscissa of convergence $\sigma \geqslant 0$. Let $K$ denote the order of this pole.

(a) If $\sigma=0$, then there is a constant $C>0$ with

$$
\pi_{\alpha}(N)=C(\log N)^{K}+\mathrm{O}\left((\log N)^{K-1}\right) .
$$

(b) If $\sigma>0$ and $K=1$, then there are constants $A, B, N_{0}>0$ such that for all $N>N_{0}$,

$$
A N^{\sigma} \leqslant \pi_{\alpha}(N) \leqslant B N^{\sigma}
$$

(c) If $\sigma>0$ and $K \geqslant 2$, then there is a constant $C>0$ with

$$
\pi_{\alpha}(N) \sim C N^{\sigma}(\log N)^{K-1}
$$

Remark 3.7 (1). If $(X, \alpha)$ is a connected system of finite combinatorial rank and $X$ has topological dimension one, the proof of Theorem 3.3 shows that $\mathrm{d}_{\alpha}$ has abscissa of convergence $\sigma=0$. Thus, the exact asymptotic of Theorem 3.6(a) applies. In general, there is no straightforward relationship between $\mathrm{d}_{\alpha}$ and the topological dimension of $X$.

Using Hlawka's bounds [6] on equidistribution in terms of discrepancy and Baker's theorem, the asymptotic in Theorem 3.6(c) could potentially be improved to give an error term.

\section{EXAMPLES}

One of the characteristic features of non-trivial $S$-integer dynamical systems is the extremely complex behaviour of their dynamical zeta functions (see [3], [4], [18, and [19] for example), so the rationality (in the sense of Theorem 3.4) of the Dirichlet series for systems of finite combinatorial rank is a little surprising.

Example 4.1. The reason for the rationality of the Dirichlet series is already visible in the simplest non-trivial example given by the map $\alpha$ dual to the map $x \mapsto 2 x$ on $\mathbb{Z}_{(3)}=\mathbb{Z}\left[\frac{1}{p} \mid p\right.$ a prime $\left.\neq 3\right]$. We have

$$
\mathrm{F}_{\alpha}(n)=\left|2^{n}-1\right|_{3}^{-1}= \begin{cases}3^{\operatorname{ord}_{3}(n)+1} & \text { if } n \text { is even } \\ 1 & \text { otherwise }\end{cases}
$$

Now

$$
\sum_{2 \nmid n} \frac{\mathrm{F}_{\alpha}(n)}{n^{z+1}}=\sum_{n \geqslant 1} \frac{1}{n^{z+1}}-\sum_{n \geqslant 1} \frac{1}{(2 n)^{z+1}}=\zeta(z+1)\left(1-\frac{1}{2^{z+1}}\right)
$$


and (on writing an even $n$ as $2 \cdot k \cdot 3^{e}$ with $3 \nmid k$ )

$$
\begin{aligned}
\sum_{2 \mid n} \frac{\mathrm{F}_{\alpha}(n)}{n^{z+1}} & =\sum_{e \geqslant 0} \sum_{k \geqslant 1,3 \nmid k} \frac{3^{e+1}}{\left(2 k 3^{e}\right)^{z+1}} \\
& =\frac{3}{2^{z+1}} \sum_{e \geqslant 0} \frac{1}{3^{e z}} \sum_{k \geqslant 1,3 \nmid k} \frac{1}{k^{z+1}} \\
& =\frac{3}{2^{z+1}} \cdot \frac{1}{1-3^{-z}} \cdot \zeta(z+1)\left(1-\frac{1}{3^{z+1}}\right) .
\end{aligned}
$$

Thus,

$$
\mathrm{d}_{\alpha}(z)=1-\frac{1}{2^{z+1}}\left(1-\frac{3}{1-3^{-z}}\left(1-\frac{1}{3^{z+1}}\right)\right)=1+\frac{1}{2^{z}}\left(\frac{1}{1-3^{-z}}\right)
$$

by (3.2). Note that the abscissa of convergence is $\sigma=0$, and this is a simple pole.

The orbit-growth function $\pi_{\alpha}$ may be obtained by extracting the coefficients from the series expression for $\mathrm{d}_{\alpha}$,

$$
\pi_{\alpha}(N)=1+\sum_{e \geqslant 0,2 \cdot 3^{e} \leqslant N} 1=1+\sum_{e=0}^{\left\lfloor\log _{3}(N / 2)\right\rfloor} 1=\frac{\log N}{\log 3}+\mathrm{O}(1) .
$$

Notice that in Example 4.1 the rational expression for $\mathrm{d}_{\alpha}$ has infinitely many singularities along the line $\Re(z)=0$, at the points $2 k \pi \mathrm{i} / \log 3$ for $k \in \mathbb{Z}$. A less direct method for obtaining an asymptotic estimate for $\pi_{\alpha}(N)$ is provided by Agmon's Tauberian theorem [1], which applies in situations like this, but as the next example shows, the general case has even worse analytic properties. This begins to illustrate the impact of additional places.

Example 4.2. Let $\alpha$ be the map dual to $x \mapsto 2 x$ on $\mathbb{Z}_{(3)} \cap \mathbb{Z}_{(5)} \subset \mathbb{Q}$. Then

$$
\mathrm{F}_{\alpha}(n)=\left|2^{n}-1\right|_{3}^{-1}\left|2^{n}-1\right|_{5}^{-1}
$$

SO

$$
\mathrm{F}_{\alpha}(n)= \begin{cases}1 & \text { if } n \text { is odd, } \\ 3 \cdot 3^{e_{1}} & \text { if } n=2 \cdot k \cdot 3^{e_{1}}, 2 \nmid k, 3 \nmid k, \\ 15 \cdot 3^{e_{1}} \cdot 5^{e_{2}} & \text { if } n=4 \cdot k \cdot 3^{e_{1}} \cdot 5^{e_{2}}, 3 \nmid k, 5 \nmid k .\end{cases}
$$

A similar calculation to that used in Example 4.1 shows that

$$
\begin{gathered}
\mathrm{d}_{\alpha}(z)=1-\frac{1}{2^{z+1}}+\frac{3}{2^{z+1}}\left(1-\frac{1}{3^{z+1}}-\frac{1}{2^{z+1}}+\frac{1}{6^{z+1}}\right) \frac{1}{1-3^{-z}} \\
+\frac{15}{4^{z+1}}\left(1-\frac{1}{3^{z+1}}-\frac{1}{5^{z+1}}+\frac{1}{15^{z+1}}\right) \frac{1}{\left(1-3^{-z}\right)\left(1-5^{-z}\right)} .
\end{gathered}
$$

Here, the abscissa of convergence $\sigma=0$ is a double pole. An asymptotic expression for $\pi_{\alpha}$ is obtained in Section 7.1

Notice that in Example 4.2, not only are there infinitely many singularities with $\Re(z)=0$, but there are singularities arbitrarily close together.

The next example illustrates the situation when a higher topological dimension is allowed. This may also be regarded as allowing places with a higher residue degree. 
Example 4.3. Let $\mathbb{K}=\mathbb{Q}(\sqrt[3]{5})$, with ring of integers

$$
\mathfrak{O}=\mathbb{Z}+\sqrt[3]{5} \mathbb{Z}+(\sqrt[3]{5})^{2} \mathbb{Z}=\mathbb{Z}[\sqrt[3]{5}]
$$

as $\mathbb{Z}$-modules. Notice that $\mathfrak{O} \cong R /\left(t^{3}-5\right)$ via the map

$$
\sqrt[3]{5} \mapsto \bar{t}=t+\left(t^{3}-5\right)
$$

There are two primes lying above the prime 2 of $\mathbb{Z}$, namely $\mathfrak{m}=\left(2,1+\bar{t}+\bar{t}^{2}\right)$ and $(2,1+\bar{t})$. The place $v$ corresponding to $\mathfrak{m}$ has residue degree 2 , since $\mathfrak{O} / \mathfrak{m} \cong \mathbb{F}_{4}$.

Let $X=\widehat{\mathfrak{O}_{\mathfrak{m}}}$ and let $\alpha$ be the map dual to $x \mapsto \sqrt[3]{5} x$. Then $X$ has topological dimension 3 , and

$$
\mathrm{F}_{\alpha}(n)=\left|\bar{t}^{n}-1\right|_{v}= \begin{cases}1 & 3 \nmid n, \\ 16 \cdot 2^{2 \operatorname{ord}_{2}(n)} & \text { otherwise. }\end{cases}
$$

In this case, the abscissa of convergence is $\sigma=1$ and this is a simple pole. Using a similar method as before (or by using (3.1) directly), we obtain the closed formula

$$
\mathrm{O}_{\alpha}(n)= \begin{cases}1 & n=1 \\ 5 & n=3 \\ 4 \cdot 2^{e} & n=3 \cdot 2^{e} \text { and } e \geqslant 1 \\ 0 & \text { otherwise }\end{cases}
$$

so

$$
\mathrm{d}_{\alpha}(z)=1+\frac{5}{3^{z}}+\frac{8}{6^{z}}\left(\frac{1}{1-2^{1-z}}\right)
$$

and

$$
\pi_{\alpha}(N)=6+4 \sum_{e=1}^{\left\lfloor\log _{2}(N / 3)\right\rfloor} 2^{e}=\frac{8}{3} 2^{-\left\{\log _{2}(N / 3)\right\}} N-2,
$$

for all $N \geqslant 6$, where $\{\cdot\}$ denotes the fractional part. Notice that (4.1) includes an oscillatory factor $\delta(N)$ appearing in Theorem 3.5 In this example,

$$
\limsup _{N \rightarrow \infty} \delta(N)=\frac{8}{3}
$$

and

$$
\liminf _{N \rightarrow \infty} \delta(N)=\frac{4}{3}
$$

To conclude this section, the following example shows that the situation is quite different for disconnected systems. Here there is no rational expression for $d_{\alpha}$ although the series organizes orbit data in a convenient way, giving an exact expression for $\pi_{\alpha}$.

Example 4.4. Let $M$ be the discrete valuation ring $\mathbb{F}_{3}[t]_{(t-1)}$ obtained by localizing the domain $\mathbb{F}_{3}[t]$ at the prime ideal $(t-1)$. Since the additive group of $M$ is torsion, $X=\widehat{M}$ is zero-dimensional. Furthermore, the map $\alpha$ dual to $x \mapsto t x$ has periodic points counted by a single place $v$ of $\mathbb{F}_{3}(t)$ corresponding to $M$. That is,

$$
\mathrm{F}_{\alpha}(n)=\left|t^{n}-1\right|_{(t-1)}^{-1}=3^{|n|_{3}^{-1}}
$$


Therefore,

$$
\begin{aligned}
\mathrm{d}_{\alpha}(z) & =\zeta(z+1)^{-1} \sum_{n \geqslant 1} \frac{3^{|n|_{3}^{-1}}}{n^{z+1}} \\
& =\zeta(z+1)^{-1} \sum_{e \geqslant 0} \frac{3^{3^{e}}}{\left(3^{e}\right)^{z+1}} \sum_{k \geqslant 1,3 \nmid k} \frac{1}{k^{z+1}} \\
& =\left(1-\frac{1}{3^{z+1}}\right) \sum_{e \geqslant 0} \frac{3^{3^{e}} / 3^{e}}{\left(3^{e}\right)^{z}}
\end{aligned}
$$

Hence, we have the following exact expression for $\pi_{\alpha}$ :

$$
\begin{aligned}
\pi_{\alpha}(N) & =\sum_{e \geqslant 0,3^{e} \leqslant N} \frac{3^{3^{e}}}{3^{e}}-\frac{1}{3} \sum_{e \geqslant 0,3^{e} \leqslant N / 3} \frac{3^{3^{e}}}{3^{e}} \\
& =1+\frac{2}{3} \sum_{e \geqslant 0,3^{e} \leqslant N} \frac{3^{3^{e}}}{3^{e}} .
\end{aligned}
$$

A further calculation shows that

$$
\limsup _{N \rightarrow \infty} \frac{N \pi_{\alpha}(N)}{3^{N}}=\frac{2}{3}
$$

and

$$
\liminf _{N \rightarrow \infty} \frac{N \pi_{\alpha}(N)}{3^{N}}=0
$$

\section{Proof of Theorems 3.3 and 3.4}

The general case requires an inclusion-exclusion argument to deal with the congruence conditions arising from multiple terms in (2.1) and multiple places in each set $P_{i}$.

Since $(X, \alpha)$ has finite combinatorial rank, the sets $P_{i}$ appearing in (2.1) are all finite. Let $T$ be the list of all the members of the sets $P_{i}$, and let $\xi_{v}$ for $v \in T$ be the image of $t$ in the appropriate field $\mathbb{K}(\mathfrak{p})$. Notice that each $\xi_{v}$ has infinite multiplicative order, and we may assume that $\left|\xi_{v}\right|_{v}=1$. For any finite non-empty sublist $S \subset T$ write

$$
f_{S}(n)=\prod_{v \in S}\left|\xi_{v}^{n}-1\right|_{v}^{-1}
$$

(notice that a given $v$ may appear in (5.1) with multiplicity). Thus $f_{T}(n)=\mathrm{F}_{\alpha}(n)$. Let $\ell_{v}$ be the multiplicative order of the image of $\xi_{v}$ in the residue field $\mathfrak{K}_{v}$ of $v$. For $S \subset T$ and a set $Q$ of rational primes, define

$$
S(Q)=\left\{v \in S \mid \operatorname{char}\left(\mathfrak{K}_{v}\right)=p \text { for some } p \in Q\right\} \subset S .
$$

If $Q=\{p\}$, we also write $S(p)$ for $S(Q)$. Consider

$$
\mathrm{d}_{\alpha}(z)=\zeta(z+1)^{-1} \sum_{n=1}^{\infty} \frac{f_{T}(n)}{n^{z+1}} .
$$

Let $\ell_{S}=\operatorname{lcm}\left\{\ell_{v} \mid v \in S\right\}$. Then the collection $\left\{N_{S}, S \subset T\right\}$, defined by

$$
N_{S}=\left\{n \in \mathbb{N}\left|\ell_{S}\right| n \text { and } \ell_{v} \nmid n \text { for all } v \in T \backslash S\right\},
$$


forms a partition of $\mathbb{N}$. Hence,

$$
\mathrm{d}_{\alpha}(z)=\zeta(z+1)^{-1} \sum_{S \subset T} \sum_{n \in N_{S}} \frac{f_{S}(n)}{n^{z+1}}
$$

since $f_{S}=f_{T}$ on $N_{S}$ by Lemma 2.6.

Let $E=\mathbb{N}_{0}^{Q}$, where

$$
Q=Q(S)=\left\{\operatorname{char}\left(\mathfrak{K}_{v}\right) \mid v \in S\right\} .
$$

For any $P \subset Q$, we extend the definition of $\varphi_{P}$ given in the statement of Theorem 3.3 to accomodate vectors $\mathbf{e}=\left(e_{p}\right) \in E$, by setting

$$
\varphi_{P}(\mathbf{e})=\prod_{p \in P} p^{e_{p}} .
$$

Each integer $n \in N_{S}$ can be written in the form

$$
n=\ell_{S} k \varphi_{Q}(\mathbf{e}),
$$

where $k \in \mathbb{N}$ satisfies

$$
p \nmid k \text { for all } p \in Q
$$

and

$$
\ell_{v} \nmid \ell_{S} k \varphi_{Q}(\mathbf{e}) \text { for all } v \in T \backslash S .
$$
Let

It is more convenient to rephrase (5.5) according to the following partition of $E$.

$$
e_{0}=\max \left\{\operatorname{ord}_{p}\left(\ell_{v}\right) \mid p \in Q, v \in T \backslash S\right\}
$$

and let $\mathbf{e}_{0} \in E$ be the vector with each entry equal to $e_{0}$. For $P \subset Q$, set

$$
E_{P}=\left\{\mathbf{e} \in E \mid e_{p} \geqslant e_{0} \text { for all } p \in P, e_{p}<e_{0} \text { for all } p \in Q \backslash P\right\},
$$

so the sets $\left\{E_{P} \mid P \subset Q\right\}$ partition $E$.

For each $q$ in the finite set $\varphi_{Q \backslash P}\left(E_{P}\right)$, let

$$
E_{P}(q)=\left\{\mathbf{e} \in E_{P} \mid \varphi_{Q \backslash P}(\mathbf{e})=q\right\} .
$$

Then, for $\mathbf{e} \in E_{P}(q)$,

$$
\ell_{S} \varphi_{Q}(\mathbf{e})=\ell_{S} \varphi_{P}(\mathbf{e}) q
$$

and if $v \in T \backslash S$,

$$
\operatorname{gcd}\left(\ell_{v}, \ell_{S} \varphi_{Q}(\mathbf{e})\right)=\operatorname{gcd}\left(\ell_{v}, \ell_{S} \varphi_{P}(\mathbf{e}) q\right)=\operatorname{gcd}\left(\ell_{v}, \ell_{S} \varphi_{P}\left(\mathbf{e}_{0}\right) q\right) .
$$

Hence, provided $\mathbf{e} \in E_{P}(q)$, condition (5.5) becomes

$$
j \nmid k \text { for all } j \in\left\{\ell_{v} / \operatorname{gcd}\left(\ell_{v}, \ell_{S} \varphi_{P}\left(\mathbf{e}_{0}\right) q\right) \mid v \in T \backslash S\right\} .
$$

For $n$ of the form (5.3), by Lemma 2.6. $f_{S}(n)=f_{S}\left(\ell_{S} \varphi_{Q}(\mathbf{e})\right)$. Furthermore, using the partition of $E$ given above, the inner sum in (5.2) may be written as

$$
\sum_{P \subset Q} \sum_{q \in \varphi_{Q \backslash P}\left(E_{P}\right)} \sum_{\mathbf{e} \in E_{P}(q)}\left(\ell_{S} \varphi_{Q}(\mathbf{e})\right)^{-z-1} f_{S}\left(\ell_{S} \varphi_{Q}(\mathbf{e})\right) \sum_{k} k^{-z-1},
$$

where $k$ runs through all natural numbers satisfying (5.4) and (5.7). Using inclusionexclusion, the inner sum in (5.8) is of the form

$$
\zeta(z+1) g\left(c^{-z}, c \in \mathcal{C}\right)
$$


where $\mathcal{C} \subset \mathbb{N}$ is a finite set of constants and $g$ is a multivariate polynomial with rational coefficients, the monomial terms of which have total degree one. Using (5.8) and (5.9), by cancelling the zeta function with its reciprocal and adjusting $\mathcal{C}$ and $g$ to absorb $\ell_{S}^{-z-1}$, (5.2) becomes

$$
\mathrm{d}_{\alpha}(z)=\sum_{S \subset T} \sum_{P \subset Q} \sum_{q \in \varphi_{Q \backslash P}\left(E_{P}\right)} \mathrm{d}_{S, P, q}(z),
$$

where

$$
\mathrm{d}_{S, P, q}(z)=g\left(c^{-z}, c \in \mathcal{C}\right) \sum_{\mathbf{e} \in E_{P}(q)} \varphi_{Q}(\mathbf{e})^{-z-1} f_{S}\left(\ell_{S} \varphi_{Q}(\mathbf{e})\right) .
$$

Again using Lemma 2.6, for each $p \in Q$, there exists $e_{p}^{\prime}$ such that for all $v \in S(p)$ and all $\mathbf{e} \in E$ with $e_{p} \geqslant e_{p}^{\prime}$,

$$
f_{v}\left(\ell_{S} \varphi_{Q}(\mathbf{e})\right)=c_{v} p^{r_{v} \omega_{v}\left(e_{p}\right)},
$$

where $c_{v}$ is a positive integer constant, $\omega_{v}\left(e_{p}\right)=e_{p}$ if the local field $\mathbb{K}_{v}$ corresponding to $v$ has zero characteristic and $\omega_{v}\left(e_{p}\right)=\eta_{v}^{\prime} p^{e_{p}}$ for some constant $\eta_{v}^{\prime} \in \mathbb{N}$, otherwise. Without loss of generality, the steps already carried out may be performed with $e_{0}$ replaced by the maximum of the value given by (5.6) and $\max \left\{e_{p}^{\prime} \mid p \in Q\right\}$.

For any $\mathbf{e} \in E_{P}(q), \varphi_{Q}(\mathbf{e})=\varphi_{P}(\mathbf{e}) q$, so the summand in (5.10) is

$$
\left(\varphi_{P}(\mathbf{e}) q\right)^{-z-1} f_{S(Q \backslash P)}\left(\ell_{S} q\right) f_{S(P)}\left(\ell_{S} \varphi_{P}(\mathbf{e})\right) .
$$

Therefore,

$$
\mathrm{d}_{S, P, q}(z)=g\left(c^{-z}, c \in \mathcal{C}\right) \sum_{\mathbf{e} \in E_{P}(q)} \varphi_{P}(\mathbf{e})^{-z-1} f_{S(P)}\left(\ell_{S} \varphi_{P}(\mathbf{e})\right),
$$

where $g$ and $\mathcal{C}$ have been adjusted to accommodate the factor

$$
q^{-z-1} f_{S(Q \backslash P)}\left(\ell_{S} q\right)
$$

appearing in (5.12). Using (5.11), it follows that

$$
\mathrm{d}_{S, P, q}(z)=C \times g\left(c^{-z}, c \in \mathcal{C}\right) \sum_{\mathbf{e} \in E_{P}(q)} \frac{\varphi_{P}(\Omega(\mathbf{e})-\mathbf{e})}{\varphi_{P}(\mathbf{e})^{z}},
$$

where $C>0$ and $\Omega: \mathbb{N}_{0}^{P} \rightarrow \mathbb{N}_{0}^{P}$ is given by

$$
\Omega(\mathbf{e})_{p}=\sum_{v \in S(p)} r_{v} \omega_{v}\left(e_{p}\right)
$$

By the definition of $E_{P}(q)$ and $\varphi_{P}$, the sum in (5.13) is

$$
\sum_{\substack{\mathbf{e} \in \mathbb{N}_{0}^{P}, e_{p} \geqslant e_{0}, p \in P}} \frac{\varphi_{P}(\Omega(\mathbf{e})-\mathbf{e})}{\varphi_{P}(\mathbf{e})^{z}}=\sum_{\mathbf{e} \in \mathbb{N}_{0}^{P}} \frac{\varphi_{P}\left(\Omega\left(\mathbf{e}+\mathbf{e}_{0}\right)-\mathbf{e}-\mathbf{e}_{0}\right)}{\varphi_{P}\left(\mathbf{e}+\mathbf{e}_{0}\right)^{z}},
$$

where $\mathbf{e}_{0} \in \mathbb{N}_{0}^{P}$ is the vector with each entry equal to $e_{0}$. For each $v \in S(p)$ with $\operatorname{char}\left(\mathbb{K}_{v}\right)=p$, set $\eta_{v}=p^{e_{0}} \eta_{v}^{\prime}$ and let $\Lambda: \mathbb{N}_{0}^{P} \rightarrow \mathbb{N}_{0}^{P}$ be defined in terms of $\eta_{v}$ as in the statement of Theorem 3.3 . Then

$$
\Omega\left(\mathbf{e}+\mathbf{e}_{0}\right)-\mathbf{e}=\Lambda(\mathbf{e})+\mathbf{d},
$$


where $\mathbf{d}=\left(d_{p}\right) \in \mathbb{N}_{0}^{P}$ is given by

$$
d_{p}=e_{0} \sum_{\substack{v \in S(p), \operatorname{char}\left(\mathbb{K}_{v}\right)=0}} r_{v} .
$$

Therefore, by (5.13) and (5.14),

$$
\mathrm{d}_{S, P, q}(z)=g\left(c^{-z}, c \in \mathcal{C}\right) \sum_{\mathbf{e} \in \mathbb{N}_{0}^{P}} \frac{\varphi_{P}(\Lambda(\mathbf{e}))}{\varphi_{P}(\mathbf{e})^{z}},
$$

where $g$ has been modified to absorb the factor $C \varphi_{P}(\mathbf{d}) \varphi_{P}\left(\mathbf{e}_{0}\right)^{-1-z}$. This concludes the proof of Theorem 3.3

To prove Theorem 3.4 first note that since $X$ is assumed to be connected, all places arise from number fields. Setting

$$
r_{S, p}=\sum_{v \in S(p)} r_{v}
$$

it follows from the definition of $\Lambda$ that

$$
\mathrm{d}_{S, P, q}(z)=g\left(c^{-z}, c \in \mathcal{C}\right) \sum_{\mathbf{e} \in \mathbb{N}_{0}^{P}} \prod_{p \in P} p^{e_{p}\left(r_{S, p}-1-z\right)} .
$$

Therefore, the sum contains a geometric series corresponding to each prime $p \in P$. Hence

$$
\mathrm{d}_{S, P, q}(z)=g\left(c^{-z}, c \in \mathcal{C}\right) \prod_{p \in P}\left(1-p^{r_{S, p}-1-z}\right)^{-1} .
$$

Since $\mathrm{d}_{\alpha}(z)$ is a finite sum of expressions of this form, this proves the theorem.

For the proof of the asymptotic results we will need a specific description of the function $g$ appearing in (5.15) in order to control cancellation between terms of different signs in various counting arguments. Notice that for a finite set of positive integers $J$ with $1 \notin J$,

$$
\sum_{I \subset J} \frac{(-1)^{|I|}}{c_{I}}>0
$$

where $c_{I}=\operatorname{lcm}\{i \mid i \in I\}$. Moreover, the construction in the proof above gives the following.

Lemma 5.1. Suppose $g \neq 0$. Then

$$
g\left(c^{-z}, c \in \mathcal{C}\right)=C \times \sum_{I \subset J} \frac{(-1)^{|I|}}{c_{I}} b_{I}^{-z},
$$

where $C>0, J$ is a finite set of positive integers with $1 \notin J$ and $b_{I}=B c_{I}$ for each $I \subset J$, for some fixed constant $B \in \mathbb{N}$.

\section{Proof of Theorem 3.5}

By Lemma 2.6, we have constants $\ell_{v}, a_{1}, \ldots, a_{d}, D$ and a prime $p$ with

$$
\mathrm{F}_{\alpha}(n)= \begin{cases}1 & \ell_{v} \nmid n, \\ a_{e} & n=\ell_{v} p^{e} k, \text { where } p \nmid k, 1 \leqslant e \leqslant d, \\ D p^{r_{v} e} & n=\ell_{v} p^{e} k, p \nmid k, e>d .\end{cases}
$$


By the method used in Section [5, we deduce that

$$
\mathrm{d}_{\alpha}(z)=1-\ell_{v}^{-z-1}+\sum_{e=1}^{d} a_{e}\left(\ell_{v} p^{e}\right)^{-z-1}+\frac{c b^{-z}}{1-p^{m-z}}\left(1-\frac{1}{p^{z+1}}\right),
$$

where $b=\ell_{v} p^{d+1}, c=\frac{D}{\ell_{v}} p^{(d+1)\left(r_{v}-1\right)}$ and $m=r_{v}-1$. It follows that, up to an error uniformly bounded in $N$, we can compute $\pi_{\alpha}(N)$ by considering the dominant term

$$
\frac{b^{-z}}{1-p^{m-z}}\left(1-\frac{1}{p^{z+1}}\right)=\frac{b^{-z}}{1-p^{m-z}}-\frac{(b p)^{-z}}{p\left(1-p^{m-z}\right)} \text {. }
$$

The first term in (6.1) contributes

$$
\sum_{e \mid b p^{e} \leqslant N} p^{m e}=\sum_{e=0}^{\left\lfloor\log _{p}(N / b)\right\rfloor} p^{m e}
$$

and the second term in (6.1) contributes

$$
\begin{aligned}
\frac{1}{p} \sum_{e \mid b p^{e+1} \leqslant N} p^{m e} & =\frac{1}{p} \sum_{e=1}^{\left\lfloor\log _{p}(N / b)\right\rfloor} p^{m(e-1)} \\
& =p^{-m-1}\left(\sum_{e=0}^{\left\lfloor\log _{p}(N / b)\right\rfloor} p^{m e}\right)-p^{-m-1} .
\end{aligned}
$$

The total contribution from (6.2) and (6.3) is therefore

$$
\left(1-p^{-m-1}\right) \sum_{e=0}^{\left\lfloor\log _{p}(N / b)\right\rfloor} p^{m e}+\mathrm{O}(1) .
$$

If $r_{v}=1$, then $m=0$ and (6.4) becomes

$$
\left(1-\frac{1}{p}\right) \log _{p}(N / b)+\mathrm{O}(1)=\frac{p-1}{p \log p} \log N+\mathrm{O}(1)
$$

If $r_{v}>1$, then $m>0$ and (6.4) becomes

$$
\begin{aligned}
C \sum_{e=0}^{\left\lfloor\log _{p}(N / b)\right\rfloor} p^{m e}+\mathrm{O}(1) & =C \cdot \frac{p^{m\left(1+\left\lfloor\log _{p}(N / b)\right\rfloor\right)}-1}{p^{m}-1}+\mathrm{O}(1) \\
& =\delta(N) N^{m}+\mathrm{O}(1)
\end{aligned}
$$

for some constant $C$ and a function $\delta$ satisfying the statement of Theorem 3.5 .

\section{Proof of Theorem 3.6}

Since $X$ is connected, $\mathrm{d}_{\alpha}$ has a rational expression (in the sense of Theorem 3.4) which is a finite sum of terms of the form (5.16). By Lemma 5.1, each such term may be written as

$$
F(z)=C \times G(z) \sum_{I \subset J} \frac{(-1)^{|I|}}{c_{I}} b_{I}^{-z}
$$

where

$$
G(z)=\prod_{p \in P}\left(1-p^{n_{p}-z}\right)^{-1}
$$


for some finite set of primes $P$ and $\mathbf{n}=\left(n_{p}\right) \in \mathbb{N}_{0}^{P}$. Let

$$
m=\max \left\{n_{p}: p \in P\right\} \text { and } L=\left|\left\{p \in P \mid n_{p}=m\right\}\right| .
$$

Note that the Dirichlet series for $G$ has abscissa of convergence $m$ and $G$ has a pole of order $L$ at $z=m$.

By showing that the coefficient of $(z-m)^{-L}$ in the Laurent series for $F$ is positive, it will follow that for at least one such $F, m=\sigma, L=K$ and that there can be no $F$ for which $m>\sigma$, nor any $F$ for which $m=\sigma$ and $L>K$.

The coefficient of $(z-m)^{-L}$ in the Laurent series for $G$ about $m$ is $\kappa=\prod_{p \in P} \kappa_{p}$, where

$$
\kappa_{p}= \begin{cases}(\log p)^{-1} & \text { if } n_{p}=m, \\ \left(1-p^{n_{p}-m}\right)^{-1} & \text { if } n_{p}<m .\end{cases}
$$

Hence, the coefficient of $(z-m)^{-L}$ in the Laurent series for $F$ is

$$
\begin{aligned}
C \kappa \sum_{I \subset J} \frac{(-1)^{|I|}}{c_{I} b_{I}^{m}} & =\frac{C \kappa}{B^{m}} \sum_{I \subset J} \frac{(-1)^{|I|}}{(\operatorname{lcm}\{i: i \in I\})^{m+1}} \\
& =\frac{C \kappa}{B^{m}} \sum_{I \subset J} \frac{(-1)^{|I|}}{\left(\operatorname{lcm}\left\{i^{m+1}: i \in I\right\}\right)} \\
& =\frac{C \kappa}{B^{m}} \sum_{I \subset J^{\prime}} \frac{(-1)^{|I|}}{(\operatorname{lcm}\{i: i \in I\})},
\end{aligned}
$$

where $J^{\prime}=\left\{j^{m+1}: j \in J\right\}$. Since the constants $\kappa, B, C$ and the result of the sum are all positive, so too is the coefficient of $(z-m)^{-L}$ in the Laurent series for $F$.

Now consider the contribution to $\pi_{\alpha}$ arising from $F$. For any $\mathbf{m} \in \mathbb{Z}^{P}$ and $x \geqslant 0$, set

$$
S_{x}^{P}(\mathbf{m})=\sum_{\substack{\mathbf{e} \in \mathbb{N}_{0}^{P}, \varphi_{P}(\mathbf{e}) \leqslant x}} \varphi_{P}(\mathbf{m e})
$$

where integer vectors are multiplied term-by-term. Extracting the coefficients from the Dirichlet series for $F$, it follows that $F$ contributes

$$
C \times \sum_{I \subset J} \frac{(-1)^{|I|}}{c_{I}} S_{N / b_{I}}^{P}(\mathbf{n})
$$

to $\pi_{\alpha}(N)$. This expression will be the main tool for obtaining our asymptotics.

The following result gives a Chebychev estimate for the individual terms $S_{N / b_{I}}^{P}(\mathbf{n})$.

Lemma 7.1. Let $\mathbf{m}=\left(m_{p}\right) \in \mathbb{Z}^{P}$ and $x \geqslant 0$. Then there exist constants $A, B$ with $B \geqslant A>0$ and $x_{0} \geqslant 0$ such that for all $x>x_{0}$,

$$
A x^{m}(\log x)^{j} \leqslant S_{x}^{P}(\mathbf{m}) \leqslant B x^{m}(\log x)^{j},
$$

where $m=\max \left\{0, m_{p}: p \in P\right\}$ and

$$
j= \begin{cases}\left|\left\{p \in P \mid m_{p}=m\right\}\right| & \text { if } m=0, \\ \left|\left\{p \in P \mid m_{p}=m\right\}\right|-1 & \text { if } m>0 .\end{cases}
$$


Proof. First suppose that $m=0$. For any $\mathbf{e} \in \mathbb{N}_{0}^{P}$,

$$
e_{p} \leqslant|P|^{-1} \log _{p} x \text { for all } p \in P \Rightarrow \varphi_{P}(\mathbf{e}) \leqslant x .
$$

Therefore,

$$
\begin{aligned}
S_{x}^{P}(\mathbf{m}) & \geqslant \prod_{p \in P} \sum_{e_{p}=0}^{\left\lfloor|P|^{-1} \log _{p} x\right\rfloor} p^{m_{p} e_{p}} \\
& \geqslant A(\log x)^{j},
\end{aligned}
$$

for some positive constant $A$. On the other hand, for any $\mathbf{e} \in \mathbb{N}_{0}^{P}$,

$$
\varphi_{P}(\mathbf{e}) \leqslant x \Rightarrow e_{p} \leqslant \log _{p} x \text { for all } p \in P .
$$

So,

$$
\begin{aligned}
S_{x}^{P}(\mathbf{m}) & \leqslant \prod_{p \in P} \sum_{e_{p}=0}^{\left\lfloor\log _{p} x\right\rfloor} p^{m_{p} e_{p}} \\
& \leqslant B(\log x)^{j},
\end{aligned}
$$

for some positive constant $B \geqslant A$.

Now suppose $m>0$. The proof is by induction on $|P|$. If $|P|=1$, then the result is obvious, so assume that $|P|>1$. Choose $q \in P$ such that $m_{q}=m$, set $P^{\prime}=P \backslash\{q\}$ and $\mathbf{m}^{\prime}=\left(m_{p}\right)_{p \in P^{\prime}}$. Write

$$
S_{x}^{P}(\mathbf{m})=\sum_{\substack{\mathbf{e} \in \mathbb{N}_{0}^{P^{\prime}} \\ \varphi_{P^{\prime}}(\mathbf{e}) \leqslant x}} \varphi_{P^{\prime}}\left(\mathbf{m}^{\prime} \mathbf{e}\right) \sum_{e_{q}=0}^{\left\lfloor\log _{q}\left(x / \varphi_{P^{\prime}}(\mathbf{e})\right\rfloor\right.} q^{m e_{q}} .
$$

The inner sum in (7.2) is a geometric series with sum

$$
\frac{q^{m\left(1+\left\lfloor\log _{q} x / \varphi_{P^{\prime}}(\mathbf{e})\right\rfloor\right)}-1}{q^{m}-1} \leqslant C_{1} x^{m} \varphi_{P^{\prime}}(-m \mathbf{e})-C_{2}
$$

for some constants $C_{1}, C_{2}>0$. It follows from (7.2) and the $m=0$ case that

$$
\begin{aligned}
S_{x}^{P}(\mathbf{m}) & \leqslant C_{1} x^{m} S_{x}^{P^{\prime}}\left(\mathbf{m}^{\prime}-m \mathbf{1}\right)-C_{2} S_{x}^{P^{\prime}}\left(\mathbf{m}^{\prime}\right) \\
& \leqslant C_{3} x^{m}(\log x)^{j}-C_{2} S_{x}^{P^{\prime}}\left(\mathbf{m}^{\prime}\right),
\end{aligned}
$$

for some constant $C_{3}>0$. Upon noting that either

- $\max \left\{m_{p}^{\prime}: p \in P^{\prime}\right\}<m$ or

- $\max \left\{m_{p}^{\prime}: p \in P^{\prime}\right\}=m$ and $\left|\left\{p \in P^{\prime} \mid m_{p}^{\prime}=m\right\}\right|-1<j$,

this completes the inductive step. A similar argument also gives the lower bound.

7.1. Proof of Theorem 3.6(a). In this case all expressions of the form (7.1) contributing to $\pi_{\alpha}(N)$ have $\mathbf{n}=\mathbf{0}$. Example 4.2 illustrates some of the issues that arise in this setting when more than one prime is involved.

Example 7.2 (Example 4.2 revisited). Let $\alpha$ be the map dual to the map $x \mapsto 2 x$ on $\mathbb{Z}_{(3)} \cap \mathbb{Z}_{(5)}$, so

$$
\mathrm{F}_{\alpha}(n)=\left|2^{n}-1\right|_{3}^{-1}\left|2^{n}-1\right|_{5}^{-1} .
$$


The term in $\mathrm{d}_{\alpha}$ that determines the asymptotic growth in $\pi_{\alpha}$ is

$$
15\left(\frac{1}{4^{z+1}}-\frac{1}{12^{z+1}}-\frac{1}{20^{z+1}}+\frac{1}{60^{z+1}}\right) \frac{1}{\left(1-3^{-z}\right)\left(1-5^{-z}\right)} .
$$

Notice that each term of the form

$$
\frac{b^{-z}}{\left(1-3^{-z}\right)\left(1-5^{-z}\right)}
$$

with $b>1$ contributes

$$
\begin{aligned}
S_{N} & =\sum_{e_{1}=1}^{\left\lfloor\log _{3}(N / b)\right\rfloor} \underbrace{\sum_{e_{2}=0}^{\left\lfloor\log _{5}\left(N / b 3^{e_{1}}\right)\right\rfloor}}_{=\frac{\log N}{\log 5}-\frac{\log 3}{\log 5} e_{1}+\mathrm{O}(1)} 1 \\
& =\frac{\log N}{\log 5} \sum_{e_{1}=1}^{\left\lfloor\log _{3}(N / b)\right\rfloor} 1-\frac{\log 3}{\log 5} \sum_{e_{1}=1}^{\left\lfloor\log _{3}(N / b)\right\rfloor} e_{1}+\mathrm{O}(\log N) \\
& =\frac{(\log N)^{2}}{\log 3 \log 5}-\frac{\log 3}{2 \log 5}\left\lfloor\log _{3}(N / b)\right\rfloor^{2}+\mathrm{O}(\log N) \\
& =\frac{(\log N)^{2}}{\log 3 \log 5}\left(1-\frac{1}{2}\right)+\mathrm{O}(\log N)
\end{aligned}
$$

to $\pi_{\alpha}(N)$. Summing over all the terms therefore gives

$$
\begin{aligned}
\pi_{\alpha}(N) & =\frac{15}{4}\left(1-\frac{1}{3}-\frac{1}{5}+\frac{1}{15}\right) \frac{(\log N)^{2}}{2 \log 3 \log 5}+\mathrm{O}(\log N) \\
& =\frac{(\log N)^{2}}{\log 3 \log 5}+\mathrm{O}(\log N) .
\end{aligned}
$$

Returning to the general case, we will use an inductive argument to obtain an exact asymptotic for $S_{x}^{P}(\mathbf{0})$. The following lemma provides the essential inductive step, but it is more general than we need at this stage (the full statement is needed for the proof of Theorem [3.6(c)). The result is a little technical and requires some preparation. Suppose that $\mathbf{m}=\left(m_{p}\right) \in \mathbb{Z}^{P}$ satisfies $m_{p} \leqslant 0$ for all $p \in P$. Set

$$
\bar{P}=\left\{p \in P: m_{p}<0\right\} .
$$

Let $\bar{P} \subseteq W \subseteq P, \mathbf{d} \in \mathbb{N}_{0}^{W}$, and define $\widetilde{\mathbf{d}}=\left(\widetilde{d}_{p}\right) \in \mathbb{N}_{0}^{W}$ by

$$
\widetilde{d}_{p}= \begin{cases}0 & \text { if } p \in \bar{P}, \\ d_{p} & \text { if } p \notin \bar{P} .\end{cases}
$$

Set

$$
T_{x}^{W}(\mathbf{m}, \mathbf{d}, k)=(\log x)^{-k} \sum_{\substack{\mathbf{e} \in \mathbb{N}_{0}^{W}, \varphi_{W}(\mathbf{e}) \leqslant x}} \varphi_{W}\left(\mathbf{m}^{\prime} \mathbf{e}\right) \mathbf{e}^{\mathbf{d}},
$$

where $\mathbf{m}^{\prime}=\left(m_{p}\right)_{p \in W}$. 
Notice that

$$
\begin{aligned}
T_{x}^{W}(\mathbf{m}, \mathbf{d}, k) & \leqslant(\log x)^{-k} \prod_{p \in W} \sum_{e_{p}=0}^{\left\lfloor\log _{p} x\right\rfloor} p^{m_{p} e_{p}} e_{p}^{d_{p}} \\
& \leqslant C(\log x)^{-k} \prod_{p \in W \backslash \bar{P}}(\log x)^{d_{p}+1} \\
& =C(\log x)^{\widetilde{\mathbf{d}} \cdot \mathbf{1}+|W \backslash \bar{P}|-k} .
\end{aligned}
$$

Lemma 7.3. Suppose that $W \neq \bar{P}$ and $W^{\prime}=W \backslash\{s\}$ for some $s \in W \backslash \bar{P}$. If $\widetilde{\mathbf{m}}=\mathbf{0}, \mathbf{d}=\widetilde{\mathbf{d}}, k=\mathbf{d} \cdot \mathbf{1}+|W \backslash \bar{P}|$, then there exist a finite indexing set $H$ and $a$ set of constants $\left\{c_{h}: h \in H\right\}$, both independent of $x$, such that

$$
T_{x}^{W}(\mathbf{m}, \mathbf{d}, k)=\left(\sum_{h \in H} c_{h} T_{x}^{W^{\prime}}\left(\mathbf{m}, \mathbf{d}(h), k_{h}\right)\right)+\mathrm{O}\left(\frac{1}{\log x}\right),
$$

where for each $h \in H, \mathbf{d}(h) \in \mathbb{N}_{0}^{W^{\prime}}$ and $k_{h} \in \mathbb{N}_{0}$ satisfy

(a) $\mathbf{d}(h)=\widetilde{\mathbf{d}(h)}$,

(b) $k_{h}=\mathbf{d}(h) \cdot \mathbf{1}+\left|W^{\prime} \backslash \bar{P}\right|$.

Proof. If $W^{\prime}=\varnothing$ the result is obvious, so assume $W^{\prime} \neq \varnothing$. By assumption $m_{s}=0$, so

$$
T_{x}^{W}(\mathbf{m}, \mathbf{d}, k)=(\log x)^{-k} \sum_{\substack{\mathbf{e} \in \mathbb{N}_{0}^{W^{\prime}}, \varphi_{W^{\prime}}(\mathbf{e}) \leqslant x}} \varphi_{W^{\prime}}\left(\mathbf{m}^{\prime} \mathbf{e}\right) \mathbf{e}^{\mathbf{d}^{\prime}} \sum_{e_{s}=0}^{\left\lfloor\log _{s}\left(x / \varphi_{W^{\prime}}(\mathbf{e})\right)\right\rfloor} e_{s}^{d_{s}}
$$

where $\mathbf{m}^{\prime}=\left(m_{p}\right)_{p \in W^{\prime}}$ and $\mathbf{d}^{\prime}=\left(d_{p}\right)_{p \in W^{\prime}}$. Expanding the inner sum,

$$
\begin{aligned}
\sum_{e_{s}=0}^{\left\lfloor\log _{s}\left(x / \varphi_{W^{\prime}}(\mathbf{e})\right)\right\rfloor} e_{s}^{d_{s}} & =C\left\lfloor\log _{s} x / \varphi_{W^{\prime}}(\mathbf{e})\right\rfloor^{d_{s}+1}+\mathrm{O}\left((\log x)^{d_{s}}\right) \\
& =C\left(\log _{s} x / \varphi_{W^{\prime}}(\mathbf{e})\right)^{d_{s}+1}+\mathrm{O}\left((\log x)^{d_{s}}\right) \\
& =C\left(\log x-\mathbf{e} \cdot(\log p)_{p \in W^{\prime}}\right)^{d_{s}+1}+\mathrm{O}\left((\log x)^{d_{s}}\right) \\
& =\sum_{h \in H} c_{h}(\log x)^{k_{h}^{\prime}} \mathbf{e}^{\mathbf{d}^{\prime}(h)}+\mathrm{O}\left((\log x)^{d_{s}}\right),
\end{aligned}
$$

where the finite indexing set $H$ and the set of constants $\left\{c_{h}: h \in H\right\}$ are both independent of $x$, and $k_{h} \in \mathbb{N}_{0}, \mathbf{d}^{\prime}(h) \in \mathbb{N}_{0}^{W^{\prime}}$ satisfy

$$
k_{h}^{\prime}+\mathbf{d}^{\prime}(h) \cdot \mathbf{1}=d_{s}+1 .
$$

Let $k_{h}=k-k_{h}^{\prime}$ and $\mathbf{d}(h)=\mathbf{d}^{\prime}+\mathbf{d}^{\prime}(h)$. Substituting (7.8) into (7.7) gives

$$
T_{x}^{W}(\mathbf{m}, \mathbf{d}, k)=\left(\sum_{h \in H^{\prime}} c_{h} T_{x}^{W^{\prime}}\left(\mathbf{m}, \mathbf{d}(h), k_{h}\right)\right)+\mathrm{O}\left(\frac{1}{\log x}\right),
$$


the error term being obtained using (7.6), since

$$
\begin{aligned}
(\log x)^{d_{s}-k} \sum_{\substack{\mathbf{e} \in \mathbb{N}_{0}^{W^{\prime}}, \varphi_{W^{\prime}}(\mathbf{e}) \leqslant x}} \varphi_{W^{\prime}}\left(\mathbf{m}^{\prime} \mathbf{e}\right) \mathbf{e}^{\mathbf{d}^{\prime}} & =\mathrm{O}\left((\log x)^{\widetilde{\mathbf{d}^{\prime}} \cdot \mathbf{1}+\left|W^{\prime} \backslash \bar{P}\right|+d_{s}-k}\right) \\
& =\mathrm{O}\left((\log x)^{\widetilde{\mathbf{d}} \cdot \mathbf{1}+|W \backslash \bar{P}|-k-1}\right) \\
& =\mathrm{O}\left((\log x)^{k-k-1}\right) .
\end{aligned}
$$

It remains to see that conditions (a) and (b) are satisfied. To see that (b) holds, note that (7.9) implies

$$
\begin{aligned}
k-k_{h}+\left(\mathbf{d}(h)-\mathbf{d}^{\prime}\right) \cdot \mathbf{1} & =d_{s}+1 \\
\Rightarrow k_{h} & =\mathbf{d}(h) \cdot \mathbf{1}+k-\mathbf{d} \cdot \mathbf{1}-1 \\
& =\mathbf{d}(h) \cdot \mathbf{1}+\left|W^{\prime} \backslash \bar{P}\right|
\end{aligned}
$$

since by hypothesis $k-\mathbf{d} \cdot \mathbf{1}=|W \backslash \bar{P}|$.

To satisfy condition (a), we show that $H$ may simply be replaced by

$$
\{h \in H: \mathbf{d}(h)=\widetilde{\mathbf{d}(h)}\}
$$

in (7.10). To see this, note that if $\mathbf{d}(h) \neq \widetilde{\mathbf{d}(h)}$, then $\widetilde{\mathbf{d}(h)} \cdot 1 \leqslant \mathbf{d}(h) \cdot \mathbf{1}-1$ and so

$$
\begin{aligned}
\widetilde{\mathbf{d}(h)} \cdot \mathbf{1}+\left|W^{\prime} \backslash \bar{P}\right|-k_{h} & \leqslant \mathbf{d}(h) \cdot \mathbf{1}+\left|W^{\prime} \backslash \bar{P}\right|-k_{h}-1 \\
& \leqslant-1,
\end{aligned}
$$

since (b) holds. Therefore,

$$
T_{x}^{W^{\prime}}\left(\mathbf{m}, \mathbf{d}(h), k_{h}\right)=\mathrm{O}\left(\frac{1}{\log x}\right)
$$

by (7.6).

Let $L=|P|$. Since

$$
(\log x)^{-L} S_{x}^{P}(\mathbf{0})=T_{x}^{W^{\prime}}(\mathbf{0}, \mathbf{0}, L),
$$

applying Lemma 7.3 and induction, we obtain

$$
(\log x)^{-L} S_{x}^{P}(\mathbf{0})=C_{1}+\mathrm{O}\left(\frac{1}{\log x}\right)
$$

for some constant $C_{1}$ which is independent of $x$. Notice that $C_{1}>0$; otherwise there would be a contradiction to the lower bound provided by Lemma 7.1. Furthermore,

$$
\begin{aligned}
S_{N / b_{I}}^{P}(\mathbf{0}) & =C_{1}\left(\log \left(N / b_{I}\right)\right)^{L}+\mathrm{O}\left(\left(\log \left(N / b_{I}\right)\right)^{L-1}\right) \\
& =C_{1}(\log N)^{L}+\mathrm{O}\left((\log N)^{L-1}\right) .
\end{aligned}
$$

It follows that the contribution to $\pi_{\alpha}(N)$ from (7.1) is

$$
C_{2}\left(\sum_{I \subset J} \frac{(-1)^{|I|}}{c_{I}}\right)(\log N)^{L}+\mathrm{O}\left((\log N)^{L-1}\right)
$$

where $C_{2}>0$ and also $\sum_{I \subset J}(-1)^{|I|} / c_{I}>0$.

Since there is at least one term of the form (17.1) contributing to $\pi_{\alpha}(N)$ for which $L=K$, this completes the proof. 
7.2. Proof of Theorem $\mathbf{3 . 6}$ (b). Recall that in this case, for each term of the form (7.1) contributing to $\pi_{\alpha}(N), \mathbf{n}=\left(n_{p}\right)$ satisfies

$$
\max \left\{n_{p}: p \in P\right\} \leqslant \sigma .
$$

Moreover, since $\sigma$ is a simple pole, any term with $\max \left\{n_{p}: p \in P\right\}=\sigma$ has at most one of the corresponding $n_{p}$ equal to $\sigma$. The upper bound now follows from Lemma 7.1

For the lower bound we use more elementary methods. The orbits for these systems mainly accumulate at specific lengths, and summing over orbits of those lengths suffices. Recall that $\mathrm{O}_{\alpha}(n)$ is related to the number of periodic points by (3.1), where $\mathrm{F}_{\alpha}(n)$ is given by the formula (5.1) with $S=T$. By (5.16), there is a prime $q \in Q$ such that

$$
\sum_{v \in U} r_{v}=\sigma+1
$$

where $U=\{v \in T|v| q\}$.

Using the notation from Section 5 let $\ell=\operatorname{lcm}\left\{\ell_{v} \mid v \in U\right\}$. Notice that we must have $\operatorname{gcd}(q, \ell)=1$ since $\ell_{v}$ divides $q^{r_{v}}-1$ for $v \in U$. Using basic properties of the the Möbius function,

$$
\begin{aligned}
\mathrm{O}_{\alpha}\left(\ell q^{e}\right)= & \frac{1}{\ell q^{e}} \sum_{e^{\prime}=0}^{e} \sum_{d \mid \ell} \mu\left(q^{e-e^{\prime}} \ell / d\right) f_{T}\left(d q^{e^{\prime}}\right) \\
= & \frac{1}{\ell q^{e}} \sum_{d \mid \ell} \mu(\ell / d)\left(f_{T}\left(d q^{e}\right)-f_{T}\left(d q^{e-1}\right)\right) \\
= & \frac{1}{\ell q^{e}}\left(f_{T}\left(\ell q^{e}\right)-f_{T}\left(\ell q^{e-1}\right)\right) \\
& \quad+\frac{1}{\ell q^{e}} \sum_{d \mid \ell, d<\ell} \mu(\ell / d)\left(f_{T}\left(d q^{e}\right)-f_{T}\left(d q^{e-1}\right)\right) .
\end{aligned}
$$

By (7.11) and Lemma 2.6. there are constants $D, E>0$ such that

$$
f_{T}\left(\ell q^{e}\right)=D q^{(\sigma+1) e} \quad \text { and } \quad f_{T}\left(d q^{e}\right) \leqslant E q^{\sigma e}
$$

for all $d<\ell$ and large enough $e$. Now,

$$
\begin{aligned}
\mathrm{O}_{\alpha}\left(\ell q^{e}\right) & \geqslant \frac{1}{\ell q^{e}}\left(\left(D q^{(\sigma+1) e}-D q^{(\sigma+1)(e-1)}\right)-2 E \ell q^{\sigma e}\right) \\
& =\frac{D}{\ell}\left(1-q^{-(\sigma+1)}\right) q^{\sigma e}-2 E q^{(\sigma-1) e},
\end{aligned}
$$

so there are constants $C_{1} \geqslant 0$ and $C_{2}, C_{3}, C_{4}>0$ such that

$$
\begin{aligned}
\pi_{\alpha}(N) & \geqslant\left(\sum_{e=0}^{\left\lfloor\log _{q} N / \ell\right\rfloor} \mathrm{O}_{\alpha}\left(\ell q^{e}\right)\right)-C_{1} \\
& \geqslant\left(\sum_{e=0}^{\left\lfloor\log _{q} N / \ell\right\rfloor} C_{2} q^{\sigma e}-C_{3} q^{(\sigma-1) e}\right)-C_{1} \\
& \geqslant C_{4} N^{\sigma},
\end{aligned}
$$

for all large $N$. 
7.3. Proof of Theorem 3.6.(c). We begin with an example that illuminates some of the issues that arise in this case.

Example 7.4. Let $M=\mathbb{Z}_{(3)}^{2} \times \mathbb{Z}_{(5)}^{2}, X=\widehat{M}$ and let $\alpha: X \rightarrow X$ be the map dual to $x \mapsto 2 x$. The dynamical system $(X, \alpha)$ has periodic point data identical to that of a product of Example 4.2 and another duplicate system. Consequently,

$$
\mathrm{F}_{\alpha}(n)= \begin{cases}1 & \text { if } n \text { is odd, } \\ 9 \cdot 3^{2 e_{1}} & \text { if } n=2 \cdot k \cdot 3^{e_{1}}, 2 \nmid k, 3 \nmid k, \\ 225 \cdot 3^{2 e_{1}} \cdot 5^{2 e_{2}} & \text { if } n=4 \cdot k \cdot 3^{e_{1}} \cdot 5^{e_{2}}, 3 \nmid k, 5 \nmid k .\end{cases}
$$

Following a similar method to that used in Example 4.2, the term of $\mathrm{d}_{\alpha}$ dominating the growth of $\pi_{\alpha}$ is

$$
\left(\frac{1}{4^{z+1}}-\frac{1}{12^{z+1}}-\frac{1}{20^{z+1}}+\frac{1}{60^{z+1}}\right) \frac{225}{\left(1-3^{1-z}\right)\left(1-5^{1-z}\right)} .
$$

Hence we need to consider

$$
S_{N / b}=\sum_{\substack{\mathbf{e} \in \mathbb{N}_{0}^{2} \\ 3^{1_{1}} 5^{e_{2}} \leqslant N / b}} 3^{e_{1}} 5^{e_{2}} .
$$

Writing $\{\cdot\}$ for the fractional part,

$$
\begin{aligned}
\frac{S_{N / b}}{N \log N} & =\frac{1}{N \log N} \sum_{\substack{\mathbf{e} \in \mathbb{N}_{0}^{2}, 3^{e_{1}} 5^{e_{2}} \leqslant N / b}} 3^{e_{1}} 5^{e_{2}} \\
& =\frac{1}{N \log N} \sum_{e_{1}=0}^{\left\lfloor\log _{3}(N / b)\right\rfloor} 3^{e_{1}} \sum_{e_{2}=0}^{\left\lfloor\log _{5}\left(N / 3^{e_{1}} b\right)\right\rfloor} 5^{e_{2}} \\
& =\frac{1}{N \log N} \sum_{e_{1}=0}^{\left\lfloor\log _{3} N / b\right\rfloor} 3^{e_{1}}\left(\frac{5 N}{4 b} 3^{-e_{1}} 5^{-\left\{\log _{5}\left(N / 3^{e_{1}} b\right)\right\}}-\frac{1}{4}\right) \\
& =\frac{5}{4 b \log N} \sum_{e_{1}=0}^{\left\lfloor\log _{3} N / b\right\rfloor} 5^{-\left\{\log _{5} N / b-e_{1} \log _{5} 3\right\}}+\mathrm{O}\left(N^{-1}\right) .
\end{aligned}
$$

Since the exponent in the inner sum is uniformly distributed in the unit interval, by Weyl's theorem [20,

$$
\begin{aligned}
\frac{1}{\log N} \sum_{e_{1}=0}^{\left\lfloor\log _{3} N / b\right\rfloor} 5^{-\left\{\log _{5} N / b-e_{1} \log _{5} 3\right\}} & \rightarrow \frac{1}{\log 3} \int_{0}^{1} 5^{-y} d y \\
& =\frac{4}{5 \log 3 \log 5} .
\end{aligned}
$$

Therefore,

$$
\frac{S_{N / b}}{N \log N} \rightarrow \frac{1}{b \log 3 \log 5}
$$


From the above and (7.12), it follows that

$$
\begin{aligned}
\frac{\pi_{\alpha}(N)}{N \log N} & \sim \frac{225}{N \log N}\left(\frac{S_{N / 4}}{4}-\frac{S_{N / 12}}{12}-\frac{S_{N / 20}}{20}+\frac{S_{N / 60}}{60}\right) \\
& \sim \frac{225}{\log 3 \log 5}\left(\frac{1}{4^{2}}-\frac{1}{12^{2}}-\frac{1}{20^{2}}+\frac{1}{60^{2}}\right) \\
& =\frac{12}{\log 3 \log 5} .
\end{aligned}
$$

In the general case, the proof uses similar ideas, with the main steps being equidistribution and an inclusion-exclusion argument. As in the previous section, for each term of the form (7.1) contributing to $\pi_{\alpha}(N), \mathbf{n}=\left(n_{p}\right)$ satisfies

$$
\max \left\{n_{p}: p \in P\right\} \leqslant \sigma
$$

and if $\max \left\{n_{p}: p \in P\right\}=\sigma$, then

$$
\left|\left\{p \in P: n_{p}=\sigma\right\}\right| \leqslant K \text {. }
$$

Furthermore, there is at least one term with

$$
\max \left\{n_{p}: p \in P\right\}=\sigma \text { and }\left|\left\{p \in P: n_{p}=\sigma\right\}\right|=K .
$$

In light of the upper bound provided by Lemma 7.1. terms of this form give the dominant contribution to $\pi_{\alpha}(N)$. Thus, to obtain the required asymptotic it suffices to prove that

$$
\frac{1}{N^{\sigma}(\log N)^{K-1}} \sum_{I \subset J} \frac{(-1)^{|I|}}{c_{I}} S_{N / b_{I}}^{P}(\mathbf{n})
$$

converges to a positive constant when $\mathbf{n}$ satisfies (7.13).

Choose a prime $q \in P$ with $n_{q}=\sigma$ and set $P^{\prime}=P \backslash\{q\}$. Write

$$
a_{N}=\frac{1}{N^{\sigma}(\log N)^{K-1}} S_{N / b}^{P}(\mathbf{n}) .
$$

Then

$$
a_{N}=\frac{1}{N^{\sigma}(\log N)^{K-1}} \sum_{\varphi_{P^{\prime}}(\mathbf{e}) \leqslant N / b} \varphi_{P^{\prime}}\left(\mathbf{n}^{\prime} \mathbf{e}\right) \sum_{e_{q}=0}^{\left\lfloor\log _{q}\left(N / b \varphi_{P^{\prime}}(\mathbf{e})\right)\right\rfloor} q^{\sigma e_{q}},
$$

where $\mathbf{n}^{\prime}=\left(n_{p}\right)_{p \in P^{\prime}}$. Treating the inner sum as a geometric progression as usual, we see that $a_{N}$ is equal to

$$
\frac{C_{1}}{b^{\sigma}(\log N)^{K-1}}\left(\sum_{\varphi_{P^{\prime}}(\mathbf{e}) \leqslant N / b} \varphi_{P^{\prime}}\left(\mathbf{n}^{\prime} \mathbf{e}\right) \varphi_{P^{\prime}}(\sigma \mathbf{e})^{-1} q^{-\sigma\left\{\log _{q}\left(N / b \varphi_{P^{\prime}}(\mathbf{e})\right)\right\}}\right)+\Delta_{N},
$$

where $C_{1}=1 /\left(1-q^{-\sigma}\right)$ and

$$
\Delta_{N}=\mathrm{O}\left(\frac{1}{N^{\sigma}(\log N)^{K-1}} S_{N / b}^{P^{\prime}}\left(\mathbf{n}^{\prime}\right)\right)=\mathrm{O}\left(\frac{1}{\log N}\right)
$$

by Lemma 7.1 .

Now let $r \in P^{\prime}$ be a prime with $m_{r}=\sigma$ (such a prime exists by hypothesis) and set $W=P \backslash\{q, r\}$. Then $a_{N}$ is, up to $\mathrm{O}(1 / \log N)$,

$$
\frac{C_{1}}{b^{\sigma}(\log N)^{K-1}} \sum_{\varphi_{W}(\mathbf{e}) \leqslant N / b} \varphi_{W}(\mathbf{m e}) \sum_{e_{r}=0}^{\left\lfloor\log _{r}\left(N / b \varphi_{W}(\mathbf{e})\right\rfloor\right.} q^{-\sigma\left\{\log _{q}\left(N / b \varphi_{W}(\mathbf{e}) r^{e_{r}}\right)\right\}}
$$


where $\mathbf{m}=\left(m_{p}\right) \in \mathbb{Z}^{W}$ is given by

$$
m_{p}= \begin{cases}n_{p}-\sigma & \text { if } n_{p}<\sigma \\ 0 & \text { if } n_{p}=\sigma\end{cases}
$$

For $\varphi_{W}(\mathbf{e}) \leqslant x$, set

$$
\mathcal{I}_{x}(\mathbf{e})=\frac{1}{\log _{r}\left(x / \varphi_{W}(\mathbf{e})\right)} \sum_{e_{r}=0}^{\left\lfloor\log _{r}\left(x / \varphi_{W}(\mathbf{e})\right)\right\rfloor} q^{-\sigma\left\{\log _{q}\left(x / \varphi_{W}(\mathbf{e}) r^{e_{r}}\right)\right\}}
$$

and

$$
\mathcal{J}_{x}(\mathbf{e})=\varphi_{W}(\mathbf{m e}) \log \left(x / \varphi_{W}(\mathbf{e})\right) .
$$

Then, again up to $\mathrm{O}(1 / \log N), a_{N}$ is

$$
\frac{C_{2}}{b^{\sigma}(\log N)^{K-1}} \sum_{\varphi_{W}(\mathbf{e}) \leqslant N / b} \mathcal{J}_{N / b}(\mathbf{e}) \mathcal{I}_{N / b}(\mathbf{e}),
$$

where $C_{2}=C_{1} / \log r$. Since $r$ and $q$ are distinct primes, the circle rotation by $\log _{q} r$ is uniquely ergodic, so there is uniform convergence for continuous functions in the ergodic theorem (see Oxtoby [10, §5] for example). Hence, given $\varepsilon>0$, there is an $M$ such that whenever $N / b \varphi_{W}(\mathbf{e}) \geqslant M$,

$$
\mathcal{I}_{N / b}(\mathbf{e})=\int_{0}^{1} q^{-\sigma y} d y+\delta_{N / b}(\mathbf{e})
$$

where $\left|\delta_{N / b}(\mathbf{e})\right|<\varepsilon$.

For $N / b \varphi_{W}(\mathbf{e})<M$ (that is, in the range $\left.N / b M<\varphi_{W}(\mathbf{e}) \leqslant N / b\right)$ there exists a uniform constant $D$ for which

$$
\mathcal{I}_{N / b}(\mathbf{e})=\int_{0}^{1} q^{-\sigma y} d y+\beta_{N / b}(\mathbf{e})
$$

where $\left|\beta_{N / b}(\mathbf{e})\right|<D$.

Let

$$
\begin{aligned}
b_{N} & =\sum_{\varphi_{W}(\mathbf{e}) \leqslant N / b} \mathcal{J}_{N / b}(\mathbf{e}), \\
c_{N} & =\sum_{\varphi_{W}(\mathbf{e}) \leqslant N / b M} \mathcal{J}_{N / b}(\mathbf{e}) \delta_{N / b}(\mathbf{e}), \text { and } \\
d_{N} & =\sum_{N / b M<\varphi_{W}(\mathbf{e}) \leqslant N / b} \mathcal{J}_{N / b}(\mathbf{e}) \beta_{N / b}(\mathbf{e}) .
\end{aligned}
$$

Then

$$
a_{N}=\frac{1}{b^{\sigma}}(\log N)^{1-K}\left(C_{3} b_{N}+C_{2} c_{N}+C_{2} d_{N}\right)+\mathrm{O}\left(\frac{1}{\log N}\right),
$$

where $C_{3}>0$ is independent of $b$.

Lemma 7.5. We have the following estimates:

(a) $(\log N)^{1-K} b_{N}$ converges to a constant $C_{4} \geqslant 0$ that is independent of $b$;

(b) $(\log N)^{1-K}\left|c_{N}\right| \leqslant C_{5} \varepsilon$;

(c) $(\log N)^{1-K} d_{N}$ converges to zero as $N \rightarrow \infty$. 
Assuming Lemma 7.5 for the moment and setting $C_{6}=C_{3} C_{4}$, we deduce that

$$
a_{N} \longrightarrow \frac{C_{6}}{b^{\sigma}} \text { as } N \rightarrow \infty \text {. }
$$

This can be used to show that (7.14) converges to a positive constant, completing the proof. To see this, first note that if $C_{6}=0$, then (7.15) contradicts the lower bound provided by Lemma 7.1, therefore $C_{6}>0$. Furthermore, (7.14) converges to

$$
\begin{aligned}
C_{6} \sum_{I \subset J} \frac{(-1)^{|I|}}{c_{I} b_{I}^{\sigma}} & =C_{6} \sum_{I \subset J} \frac{(-1)^{|I|}}{c_{I}\left(B c_{I}\right)^{\sigma}} \\
& =\frac{C_{6}}{B^{\sigma}} \sum_{I \subset J^{\prime}} \frac{(-1)^{|I|}}{\operatorname{lcm}\{i \mid i \in I\}} \\
& >0,
\end{aligned}
$$

where $J^{\prime}=\left\{i^{\sigma+1} \mid i \in J\right\}$.

Finally, we turn to the postponed proof of the lemma.

Proof of Lemma 7.5. To prove (a), first note that we may write

$$
\begin{aligned}
(\log N)^{1-K} b_{N} & =\frac{1}{(\log (N / b)+\log b)^{K-1}} b_{N} \\
& =\left(1+\Delta_{N}\right)^{-1}(\log (N / b))^{1-K} b_{N}
\end{aligned}
$$

where $\Delta_{N}=\mathrm{O}\left((\log N)^{-1}\right)$. Therefore, it suffices to prove that

$$
(\log (N / b))^{1-K} b_{N} \rightarrow C_{4}
$$

Write $x=N / b$ and notice that

$$
\begin{aligned}
(\log (N / b))^{1-K} b_{N} & =(\log x)^{1-K} \sum_{\varphi_{W}(\mathbf{e}) \leqslant x} \mathcal{J}_{x}(\mathbf{e}) \\
& =(\log x)^{1-K} \sum_{\varphi_{W}(\mathbf{e}) \leqslant x} \varphi_{W}(\mathbf{m e}) \log \left(x / \varphi_{W}(\mathbf{e})\right) \\
& =(\log x)^{1-K} \sum_{\varphi_{W}(\mathbf{e}) \leqslant x} \varphi_{W}(\mathbf{m e})\left(\log x-\mathbf{e} \cdot(\log p)_{p \in W}\right) .
\end{aligned}
$$

Therefore, $(\log (N / b))^{1-K} b_{N}$ may be written as a sum of $|W|+1$ expressions of the form

$$
(\log x)^{-k} \sum_{\varphi_{W}(\mathbf{e}) \leqslant x} \varphi_{W}(\mathbf{m e}) \mathbf{e}^{\mathbf{d}}
$$

where $\mathbf{d} \in \mathbb{N}_{0}^{W}$ satisfies

$$
k-\mathbf{d} \cdot \mathbf{1}=K-2=|W \backslash \bar{P}|,
$$

with $\bar{P}$ defined by (7.3).

So, we must consider an expression of the form $T_{x}^{W}(\mathbf{m}, \mathbf{d}, k)$, defined by $(7.5)$. Let $\widetilde{\mathbf{d}}$ be defined by (7.4). If $\mathbf{d} \neq \widetilde{\mathbf{d}}$, then $\widetilde{\mathbf{d}} \cdot \mathbf{1}<\mathbf{d} \cdot \mathbf{1}$. Hence by $(7.6)$ and (7.16),

$$
T_{x}^{W}(\mathbf{m}, \mathbf{d}, k)=\mathrm{O}\left(\frac{1}{\log x}\right) .
$$


Thus, we need only consider those terms $T_{x}^{W}(\mathbf{m}, \mathbf{d}, k)$ with $\mathbf{d}=\widetilde{\mathbf{d}}$. If $\bar{P}=\varnothing$, then proceeding as in Section 7.1, an induction using Lemma 7.3 shows that

$$
T_{x}^{W}(\mathbf{m}, \mathbf{d}, k)=C_{7}+\mathrm{O}\left(\frac{1}{\log x}\right)
$$

where $C_{7}$ is a constant independent of $x$. If $\bar{P} \neq \varnothing$, then again by induction using Lemma 7.3, up to an error of $\mathrm{O}(1 / \log x), T_{x}^{W}(\mathbf{m}, \mathbf{d}, k)$ may be written as a linear combination of expressions of the form

$$
T_{x}^{\bar{P}}(\mathbf{m}, \mathbf{0}, 0)=\sum_{\varphi \bar{P}(\mathbf{e}) \leqslant x} \varphi \bar{P}\left(\mathbf{m}^{\prime} \mathbf{e}\right)
$$

where $\mathbf{m}^{\prime}=\left(m_{p}\right)_{p \in \bar{P}}$. Moreover,

$$
\prod_{p \in \bar{P}} \sum_{p=0}^{\left\lfloor|\bar{P}|^{-1} \log _{p} x\right\rfloor} p^{m_{p} e_{p}} \leqslant T_{x}^{\bar{P}}(\mathbf{m}, \mathbf{0}, 0) \leqslant \prod_{p \in \bar{P}} \sum_{p=0}^{\left\lfloor\log _{p} x\right\rfloor} p^{m_{p} e_{p}}
$$

SO

as $x \rightarrow \infty$.

$$
T_{x}^{\bar{P}}(\mathbf{m}, \mathbf{0}, 0) \longrightarrow \prod_{p \in \bar{P}} \frac{1}{1-p^{m_{p}}}
$$

Thus, $(\log (N / b))^{1-K} b_{N}$ converges to a constant $C_{4}$ independent of $b$ which is non-negative, as each term of the sequence is non-negative.

To prove (b), simply note that $\left|c_{N}\right| \leqslant b_{N} \varepsilon$. To prove (c), first notice that

$$
\begin{aligned}
\left|d_{N}\right| & \leqslant D \sum_{N / b M<\varphi_{W}(\mathbf{e}) \leqslant N / b} \mathcal{J}_{N / b}(\mathbf{e}) \\
& =D\left(b_{N}-\sum_{\varphi_{W}(\mathbf{e}) \leqslant N / b M} \mathcal{J}_{N / b}(\mathbf{e})\right) .
\end{aligned}
$$

Finally,

$$
\mathcal{J}_{N / b}(\mathbf{e})=\mathcal{J}_{N / b M}(\mathbf{e})+(\log M) \varphi_{W}(\mathbf{m e})
$$

SO

$$
\begin{aligned}
(\log N)^{1-K}\left|d_{N}\right| \leqslant & D(\log N)^{1-K}\left(b_{N}-b_{N / M}\right) \\
& -D(\log M)(\log N)^{1-K} S_{N / b M}^{W}(\mathbf{m}) \\
= & D(\log N)^{1-K}\left(b_{N}-b_{N / M}\right)+\mathrm{O}\left(\frac{1}{\log N}\right),
\end{aligned}
$$

by Lemma 7.1. Since $(\log N)^{1-K} b_{N}$ and $(\log N)^{1-K} b_{N / M}$ both converge to $C_{4}$, (c) follows.

\section{REFERENCES}

[1] S. Agmon, Complex variable Tauberians, Trans. Amer. Math. Soc. 74 (1953), 444-481. MR0054079 (14,869a)

[2] V. Chothi, G. Everest, and T. Ward, S-integer dynamical systems: Periodic points, J. Reine Angew. Math. 489 (1997), 99-132. MR1461206 (99b:11089)

[3] G. Everest, R. Miles, S. Stevens, and T. Ward, Orbit-counting in non-hyperbolic dynamical systems, J. Reine Angew. Math. 608 (2007), 155-182. MR2339472 (2008k:37042) 
[4] G. Everest, V. Stangoe, and T. Ward, Orbit counting with an isometric direction, Algebraic and topological dynamics, Contemp. Math., vol. 385, Amer. Math. Soc., Providence, RI, 2005, pp. 293-302. MR2180241 (2006k:37046)

[5] G. H. Hardy and M. Riesz, The general theory of Dirichlet's series, Cambridge Tracts in Mathematics and Mathematical Physics, No. 18, Stechert-Hafner, Inc., New York, 1964. MR0185094 (32:2564)

[6] E. Hlawka, Discrepancy and uniform distribution of sequences, Compositio Math. 16 (1964), 83-91 (1964). MR0174544 (30:4745)

[7] D. A. Lind and T. Ward, Automorphisms of solenoids and p-adic entropy, Ergodic Theory Dynam. Systems 8 (1988), no. 3, 411-419. MR961739 (90a:28031)

[8] R. Miles, Zeta functions for elements of entropy rank-one actions, Ergodic Theory Dynam. Systems 27 (2007), no. 2, 567-582. MR2308145 (2008h:37008)

[9] _ Periodic points of endomorphisms on solenoids and related groups, Bull. Lond. Math. Soc. 40 (2008), no. 4, 696-704. MR2441142

[10] J. C. Oxtoby, Ergodic sets, Bull. Amer. Math. Soc. 58 (1952), 116-136. MR0047262(13,850e)

[11] W. Parry, An analogue of the prime number theorem for closed orbits of shifts of finite type and their suspensions, Israel J. Math. 45 (1983), no. 1, 41-52. MR.710244 (85c:58089)

[12] W. Parry and M. Pollicott, An analogue of the prime number theorem for closed orbits of Axiom A flows, Ann. of Math. (2) 118 (1983), no. 3, 573-591. MR727704 (85i:58105)

[13] Z Z Z Z _ functions and the periodic orbit structure of hyperbolic dynamics, Astérisque (1990), no. 187-188, 268. MR1085356 (92f:58141)

[14] D. Ruelle, Dynamical zeta functions and transfer operators, Notices Amer. Math. Soc. 49 (2002), no. 8, 887-895. MR 1920859 (2003d:37026)

[15] V. Stangoe, Orbit counting far from hyperbolicity, Ph.D. thesis, University of East Anglia, 2004.

[16] R. P. Stanley, Enumerative combinatorics. Vol. 1, Cambridge Studies in Advanced Mathematics, vol. 49, Cambridge University Press, Cambridge, 1997, with a foreword by Gian-Carlo Rota, corrected reprint of the 1986 original. MR 1442260 (98a:05001)

[17] S. Waddington, The prime orbit theorem for quasihyperbolic toral automorphisms, Monatsh. Math. 112 (1991), no. 3, 235-248. MR.1139101(92k:58219)|

[18] T. Ward, Almost all S-integer dynamical systems have many periodic points, Ergodic Theory Dynam. Systems 18 (1998), no. 2, 471-486. MR1619569 (99k:58152)

[19] — Dynamical zeta functions for typical extensions of full shifts, Finite Fields Appl. 5 (1999), no. 3, 232-239. MR.1702897 (2000m:11067)

[20] H. Weyl, Über die Gleichverteilung von Zahlen mod. Eins, Math. Ann. 77 (1916), no. 3, 313-352. MR1511862

School of Mathematics, University of East Anglia, Norwich NR4 7TJ, United KingDOM

E-mail address: g.everest@uea.ac.uk

Department of Mathematics, KTH-Royal Institute of Technology, SE-100 44 StockHOLM, SWEDEN

E-mail address: ricmiles@kth.se

School of Mathematics, University of East Anglia, Norwich NR4 7TJ, United KingDOM

E-mail address: shaun.stevens@uea.ac.uk

School of Mathematics, University of East Anglia, Norwich NR4 7TJ, United KingDOM

E-mail address: t.ward@uea.ac.uk 